\title{
Upscaling of dislocation walls in finite domains
}

\author{
P. VAN MEURS ${ }^{1,3}$, A. MUNTEAN ${ }^{1,2}$ and M. A. PELETIER ${ }^{1,2}$ \\ ${ }^{1}$ Centre for Analysis, Scientific computing and Applications (CASA), \\ Department of Mathematics and Computer Science, \\ Eindhoven University of Technology, \\ P.O. Box 513, \\ 5600 MB Eindhoven, The Netherlands \\ 2 Institute for Complex Molecular Systems (ICMS), \\ Eindhoven University of Technology, \\ 3 email: p.j.p.v.meurs@tue.nl (corresponding author)
}

October 16, 2018

\begin{abstract}
We wish to understand the macroscopic plastic behaviour of metals by upscaling the micro-mechanics of dislocations. We consider a highly simplified dislocation network, which allows our microscopic model to be a one dimensional particle system, in which the interactions between the particles (dislocation walls) are singular and non-local.

As a first step towards treating realistic geometries, we focus on finite-size effects rather than considering an infinite domain as typically discussed in the literature. We derive effective equations for the dislocation density by means of $\Gamma$-convergence on the space of probability measures. Our analysis yields a classification of macroscopic models, in which the size of the domain plays a key role.
\end{abstract}

Key Words: Plasticity; Multiscale; Straight edge-dislocations; Discrete-to-continuum limit; Г-convergence; 74Q05, 74C05, 82B21, 49J45, 82D35

\section{Introduction}

Dislocations in metals are curve-like defects in the atomic lattice of the metal. Typical metals have many dislocations (as much as $1000 \mathrm{~km}$ of dislocation curve in a cubic millimeter [23, p. 20]), and their collective motion is the microscopic mechanism behind macroscopic permanent or plastic deformation.

At scales of millimeters or more, plastic deformation is well described by continuumlevel theories (see e.g. [3, Ch.6]); at scales of 1-100 $\mu \mathrm{m}$, however, the specimen size, material grain size, and dislocation distribution scales become comparable, and these high-level theories break down. At these smaller scales, crystal plasticity models attempt to capture the interaction between dislocations and grain boundaries by including additional degrees of freedom representing dislocation densities. 
Although more detailed, such (meso-scale) crystal-plasticity models depend on closing the BBGKY hierarchy of multi-point correlation functions at the two-point or three-point correlation levels. Current methods commonly postulate a closure assumption involving certain averages, and estimate these averages from the statistics of smaller-scale models. see for instance [35, 17, 33, 5, 25, 6.

While this statistical approach makes sense from a practical point of view, the question remains whether microscopic models of dislocations could not be scaled up rigorously, without ad hoc closure assumptions - although possibly in a simpler setup. Such a rigorous upscaling has been performed, for instance, for the case of parallel dislocations on a single slip plane, represented by a 'queue' of points on the real line [14, 10, 11, 12, 18, for arbitrary planar dislocations 24, 1, and for arbitrary collections of parallel dislocations [4, 27, 13.

However, these upscaling techniques fail to capture one of the more intriguing aspects of interaction dislocations: the cancellation that takes place in pile-ups of edge dislocations at grain boundaries. Roy et al. pointed out 29] that the stresses in such pile-ups are very sensitive to the local stacking of the dislocations, leading to incorrect predictions if the averaging is not done correctly. This may also be the reason why there are multiple, mutually contradicting dislocation-density models in the literature (e.g. [16, 17, 9]).

Sparked by this observation, Scardia et al. analyzed the structure of pile-ups in detail [15, 30, and showed that five different regimes exist, depending on a local aspect ratio (see also [19, 21, 32] for an analysis of one of these regimes using formal asymptotics). We describe the results of [15, 30] in detail below.

The authors of [15, 30] made several simplifying assumptions, one of which is to allow the dislocations to move in a half-infinite domain. Since dislocation-density models aim to describe the cases where grain size and pile-up width are comparable, a finite domain bounded by grain boundaries on both sides is more natural. In this paper, we therefore generalize the results of [15, 30] by considering any finite length for the domain in which the dislocations are situated. This brings us to our main research question:

How do finite domains change the results from [15, 30]?

After introducing our microscopic model (Section 1.1), we describe the upscaling procedure in Section 1.2. Then we state Theorem 1.1- our main result - and how to interpret it from a practical point of view.

\subsection{Setting of the microscopic energy}

Inspired by [29], we consider the dislocations to be arranged equidistantly in $n+1$ vertical walls of dislocations, which are assumed to be infinitely long. Figure 1 shows a schematic picture of this configuration.

In the steady state, we obtain the positions of the dislocation walls, denoted by $\tilde{x}_{1} \leq$ $\tilde{x}_{2} \leq \ldots \leq \tilde{x}_{n}$, by minimizing the energy given by

$$
\mathcal{E}=\mathcal{E}^{(\mathrm{i})}+\mathcal{E}^{(\mathrm{F})}+\mathcal{E}^{(\mathrm{L})},
$$




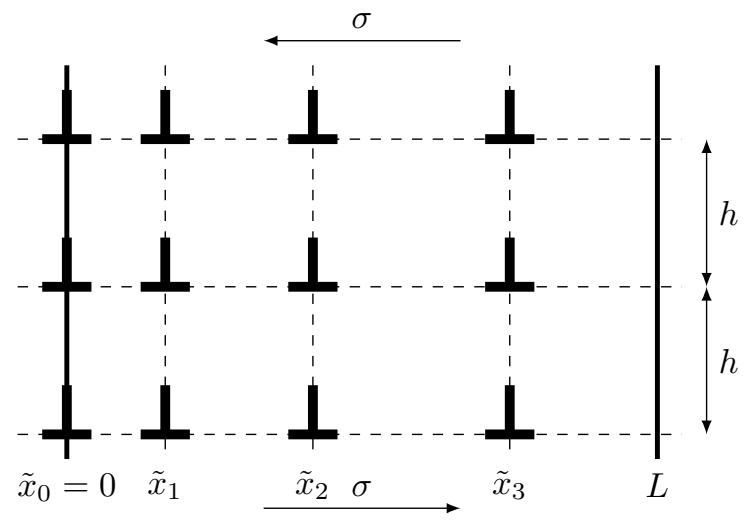

Figure 1. Configuration of dislocations in the microscopic model.

$$
\begin{aligned}
& \mathcal{E}^{(\mathrm{i})}(\mathbf{x})=K \sum_{k=1}^{n} \sum_{j=0}^{n-k} V\left(\pi \frac{\tilde{x}_{j+k}-\tilde{x}_{j}}{h}\right), \\
& \mathcal{E}^{(\mathrm{F})}(\mathbf{x})=\sigma \sum_{i=1}^{N} \tilde{x}_{i}, \\
& \mathcal{E}^{(\mathrm{L})}(\mathbf{x})= \begin{cases}0, & \text { if } \tilde{x}_{n} \leq L, \\
\infty, & \text { otherwise. }\end{cases}
\end{aligned}
$$

Here, $V$ is the interaction potential between walls, which is defined by

$$
V(r):=r \operatorname{coth} r-\log |\sinh r|-\log 2, r \in \mathbb{R} .
$$

The potential $V$ is even, has a logarithmic singularity at the origin, and is strictly convex and monotonic on $(-\infty, 0)$ and $(0, \infty)$. The energy $\mathcal{E}$ involves five model parameters: $n$, the number of walls minus $1 ; h$, the distance between two subsequent dislocations in a wall; $\sigma$, a constant external load applied to the system; $L$, the position of the right boundary; $K$, a material constant.

Let us explain our model in terms of the expression for $\mathcal{E}(\mathbf{x})$. The interaction part $\mathcal{E}^{(\mathrm{i})}$ is minimized by spreading the walls far apart in the interval $(0, \infty)$. The 0 is due to the pinned wall at the impenetrable barrier located at $\tilde{x}_{0}=0$. Due to the logarithmic singularity of $V$ at 0 , none of the other walls will be located at $\tilde{x}_{0}$. The parts coming from the external load $\mathcal{E}^{(\mathrm{F})}$ and from the right impenetrable barrier $\mathcal{E}^{(\mathrm{L})}$ are minimized by putting the walls close to 0 . The unique minimizer (see Proposition 2.3) of $\mathcal{E}$ balances these effects. A thorough understanding of this balance will explain how the finiteness of the domain changes the results from [15].

\subsection{Upscaling}

As mentioned in the introduction, the collective behaviour of dislocation walls will be obtained by scaling up the system described above, resulting in an energy functional $E$ which depends only on a dislocation density $\mu$. For this we need to define what it means for $\mu$ to be "close to" a vector $\tilde{\mathbf{x}}$ of discrete wall positions. We do this by using the narrow 
topology. Setting

$$
\mu_{n}=\frac{1}{n} \sum_{j=1}^{n} \delta_{\tilde{x}_{j}} .
$$

we say that $\mu_{n}$ converges in the narrow topology to $\mu$ if and only if

$$
\int_{[0, \infty)} \varphi d \mu_{n} \rightarrow \int_{[0, \infty)} \varphi d \mu \quad \text { for all } \varphi \in C_{b}([0, \infty)) .
$$

As $V$ has a logarithmic singularity at 0 , the energy landscape of $\mathcal{E}$ contains $\mathcal{O}(n)$ singularities. Hence $\mathcal{E}$ will never be close to any limiting energy $E$ in any $L^{\infty}$-topology. Instead, we aim to prove that $\mathcal{E} \Gamma$-converges to $E$ provided that an appropriate scaling is applied. With $\Gamma$-convergence, we can show that the minimizer of $\mathcal{E}$ is close to a minimizer of $E$. Furthermore, $\Gamma$-convergence is robust to a perturbation by a continuous functional (which may model another type of external force term, for example).

From now on, all the parameters $(L, h, K, \sigma)$ depend on $n$. In order to obtain a meaningful limit we rescale the positions $\mathbf{x}$ and the energy $\mathcal{E}$ in some $n$-dependent manner. There are two natural length scales for the rescaling of $\mathbf{x}$, one given by the size $L_{n}$ of the domain, and the other provided by an intrinsic scale arising from the balance between the load parameter $\sigma_{n}$ and the interaction term $\mathcal{E}^{(\mathrm{i})}$.

Inspired by [15] we define this second length scale as

$$
\ell_{n}=\frac{n h_{n}}{\pi} \hat{\alpha}_{n}
$$

where $\hat{\alpha}_{n}$ is a parameter which scales like the aspect ratio between the dislocations in Figure 1, i.e. the typical horizontal distance between walls divided by $h_{n}$. It depends on the parameters in the following way:

$$
\begin{aligned}
& \hat{\alpha}_{n}:=f_{n}\left(\sqrt{\frac{\pi K_{n}}{n \sigma_{n} h_{n}}}\right), \text { with } \\
& f_{n}(a):= \begin{cases}n a^{2}, & a<\frac{1}{n}, \\
a, & \frac{1}{n} \leq a \leq 1, \\
\log a+1, & 1<a .\end{cases}
\end{aligned}
$$

Figure 2 illustrates the typical behaviour of $f_{n}$. We define the ratio

$$
\gamma_{n}:=\frac{L_{n}}{\ell_{n}}
$$

to characterize the relative size of $\ell_{n}$ and $L_{n}$.

Whenever $\ell_{n}$ is asymptotically smaller than $L_{n}$, i.e. $\gamma_{n} \gg 1$, it is natural to rescale the positions by $\ell_{n}$. In this case the scaled energy is given by

$$
E_{n}\left(\mathbf{x}^{n}\right):= \begin{cases}\frac{1}{n \sigma_{n} \ell_{n}} \mathcal{E}\left(\ell_{n} \mathbf{x}^{n}\right)-\frac{1}{2} \log \frac{e}{2 n \hat{\alpha}_{n}}, & \text { if } \hat{\alpha}_{n} \ll 1 / n, \\ \frac{1}{n \sigma_{n} \ell_{n}} \mathcal{E}\left(\ell_{n} \mathbf{x}^{n}\right), & \text { otherwise. }\end{cases}
$$

The $\Gamma$-convergence result of $E_{n}$ to $E$ is stated in [15, Theorem 1]. There are five expres- 


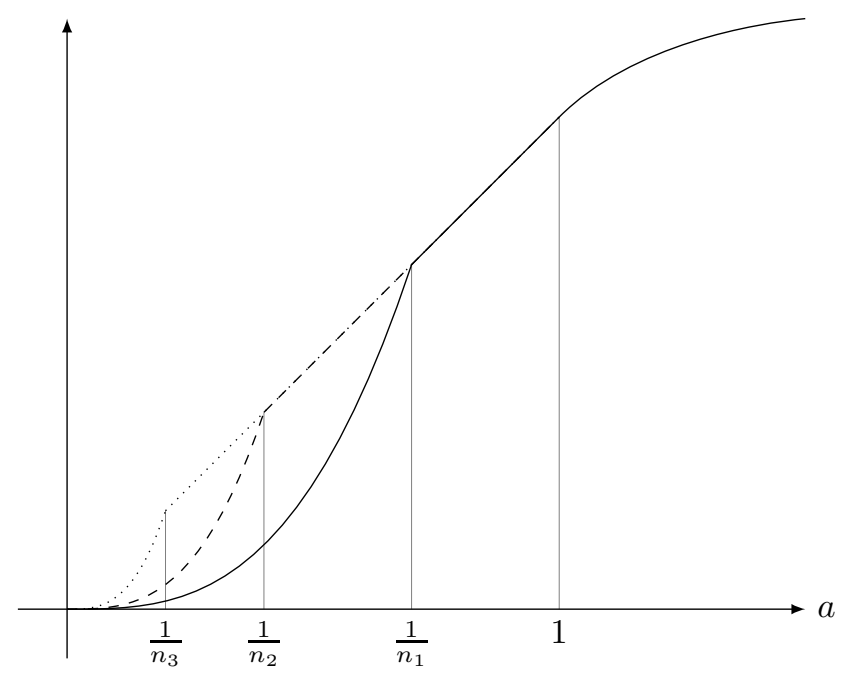

Figure 2. Plots of $f_{n}$ (see (1.5)) for $n_{1}<n_{2}<n_{3}$.

sions for the related limiting energy $E$, depending on which of five scaling regimes $\hat{\alpha}_{n}$ belongs to. We come back to this while discussing Table 1.

On the other hand, when $L_{n} \lesssim \ell_{n}$, i.e. $\gamma_{n} \lesssim 1$, the barrier at $L_{n}$ is likely to determine the typical length scale for $\tilde{\mathbf{x}}$, and we scale $\tilde{\mathbf{x}}$ with $L_{n}$. The expression for the aspect ratio then also changes:

$$
\alpha_{n}:=\frac{\pi L_{n}}{n h_{n}}=\gamma_{n} \hat{\alpha}_{n} .
$$

In this case (i.e. $\gamma_{n} \ll 1$ or $\gamma_{n} \sim 1$ ), we scale the energy as follows:

$$
E_{n}\left(\mathbf{x}^{n}\right):= \begin{cases}\frac{\gamma_{n}}{n \sigma_{n} L_{n}} \mathcal{E}\left(L_{n} \mathbf{x}^{n}\right)-\frac{1}{2} \log \frac{e}{2 n \alpha_{n}}, & \text { if } \alpha_{n} \ll 1 / n, \\ \frac{\exp \left(2 \alpha_{n}\left(1-1 / \gamma_{n}\right)\right)}{n \sigma_{n} L_{n}} \mathcal{E}\left(L_{n} \mathbf{x}^{n}\right), & \text { if } \alpha_{n} \gg 1, \\ \frac{\gamma_{n}^{2}}{n \sigma_{n} L_{n}} \mathcal{E}\left(L_{n} \mathbf{x}^{n}\right), & \text { otherwise. }\end{cases}
$$

In order to state the main result we extend $E_{n}$ to apply to measures by setting

$$
E_{n}(\mu)= \begin{cases}E_{n}\left(\mathbf{x}^{n}\right), & \text { if } \mu=\frac{1}{n} \sum_{j=1}^{n} \delta_{x_{j}} \\ \infty, & \text { otherwise }\end{cases}
$$

Theorem 1.1 (Convergence of the energy). Let $\alpha_{n}$ and $\gamma_{n}$ be such that they satisfy any of the criteria as in the first columns of Table 1 and Table 2 . Then boundedness of $E_{n}\left(\mu_{n}\right)$ (as in (1.10) implies that $\left(\mu_{n}\right)$ is compact in the narrow topology. Moreover, $E_{n}$ $\Gamma$-converges with respect to the narrow topology to

$$
E=E^{(\mathrm{i})}+E^{(\mathrm{F})}+E^{(\mathrm{L})},
$$


Table 1. Expressions for $E^{(\mathrm{i})}$, the interaction part of the limit energy. If $\gamma_{n} \gg 1$, one has to read $\hat{\alpha}_{n}$ instead of $\alpha_{n}$.

\begin{tabular}{|c|c|c|}
\hline regime & $E^{(\mathrm{i})}(\mu)$ & $p$ \\
\hline$\alpha_{n} \ll \frac{1}{n}$ & $\frac{1}{2} \int_{0}^{\infty} \int_{0}^{\infty} \log \frac{1}{|x-y|} d \mu(y) d \mu(x)$ & 1 \\
\hline$n \alpha_{n} \rightarrow \tilde{c}$ & $\frac{c}{2} \int_{0} \int_{0} V(\tilde{c}(x-y)) d \mu(y) d \mu(x)$ & 2 \\
\hline$\frac{1}{n} \ll \alpha_{n} \ll 1$ & $\begin{cases}\left(\int_{0}^{\infty} V\right) \int_{0}^{\infty} \rho^{2}, & \text { if } d \mu(x)=\rho(x) d x \\
\infty, & \text { otherwise }\end{cases}$ & 3 \\
\hline$\alpha_{n} \rightarrow \tilde{c}$ & $\begin{cases}\tilde{c} \int_{0}\left(\sum_{k=1} V\left(k \frac{c}{\rho(x)}\right)\right) \rho(x) d x, & \text { if } d \mu(x)=\rho(x) d x \\
\infty, & \text { otherwise }\end{cases}$ & 4 \\
\hline $1 \ll \alpha_{n}$ & $\begin{cases}2 e^{-2} \mathbb{1}_{\{\gamma \leq 1\}}, & \text { if } \frac{d \mu}{d \mathcal{L}}=\rho \leq 1 \quad \mathcal{L} \text {-a.e. } \\
\infty, & \text { otherwise }\end{cases}$ & 5 \\
\hline
\end{tabular}

Table 2. Expressions for $E^{(\mathrm{F})}$ and $E^{(\mathrm{L})}$, the parts in the limit energy coming from the external force and the second barrier. The constant $C$ is given by (1.11).

\begin{tabular}{|c|c|c|c|}
\hline regime & $E^{(\mathrm{F})}(\mu)$ & $E^{(\mathrm{L})}(\mu)$ & $q$ \\
\hline$\gamma_{n} \gg 1$ & $\int_{0}^{\infty} x d \mu(x)$ & 0 & 1 \\
\hline$\gamma_{n} \rightarrow \gamma$ & $C\left(\gamma ;\left(\alpha_{n}\right)\right) \int_{0}^{\infty} x d \mu(x)$ & $\begin{cases}0, & \text { if } \operatorname{supp} \mu \subset[0,1] \\
\infty, & \text { otherwise. }\end{cases}$ & 2 \\
\hline$\gamma_{n} \ll 1$ & 0 & $\begin{cases}0, & \text { if } \operatorname{supp} \mu \subset[0,1], \\
\infty, & \text { otherwise. }\end{cases}$ & 3 \\
\hline
\end{tabular}

where the components are given in Table 11 and Table 2, except for the particular case in which $1 \ll \alpha_{n}$ and $\exp \left(2 \alpha_{n}\left(1-1 / \gamma_{n}\right)\right) \rightarrow \infty$, which is treated in Theorem 4.1.

The state of the art before this paper is given by Table 1 Table 2 shows our generalization of the results of [15] to finite domains. For a given set of parameters $\left(n, L_{n}, h_{n}\right.$, $K_{n}, \sigma_{n}$ ), we can calculate $\ell_{n}$ and consecutively $\gamma_{n}$ and $\alpha_{n}$, and thus we know a priori which of the expressions for $E^{(\mathrm{i})}(\mu), E^{(\mathrm{F})}(\mu)$ and $E^{(\mathrm{L})}(\mu)$ we have as limit energy.

In all cases the limit energy gives rise to a well-posed variational problem: minimizers exist and are unique (Theorem 4.2). By the usual results on $\Gamma$-convergence, minimizers are the limit of the sequence of the finite- $n$ minimizers (Corollary 4.3). 


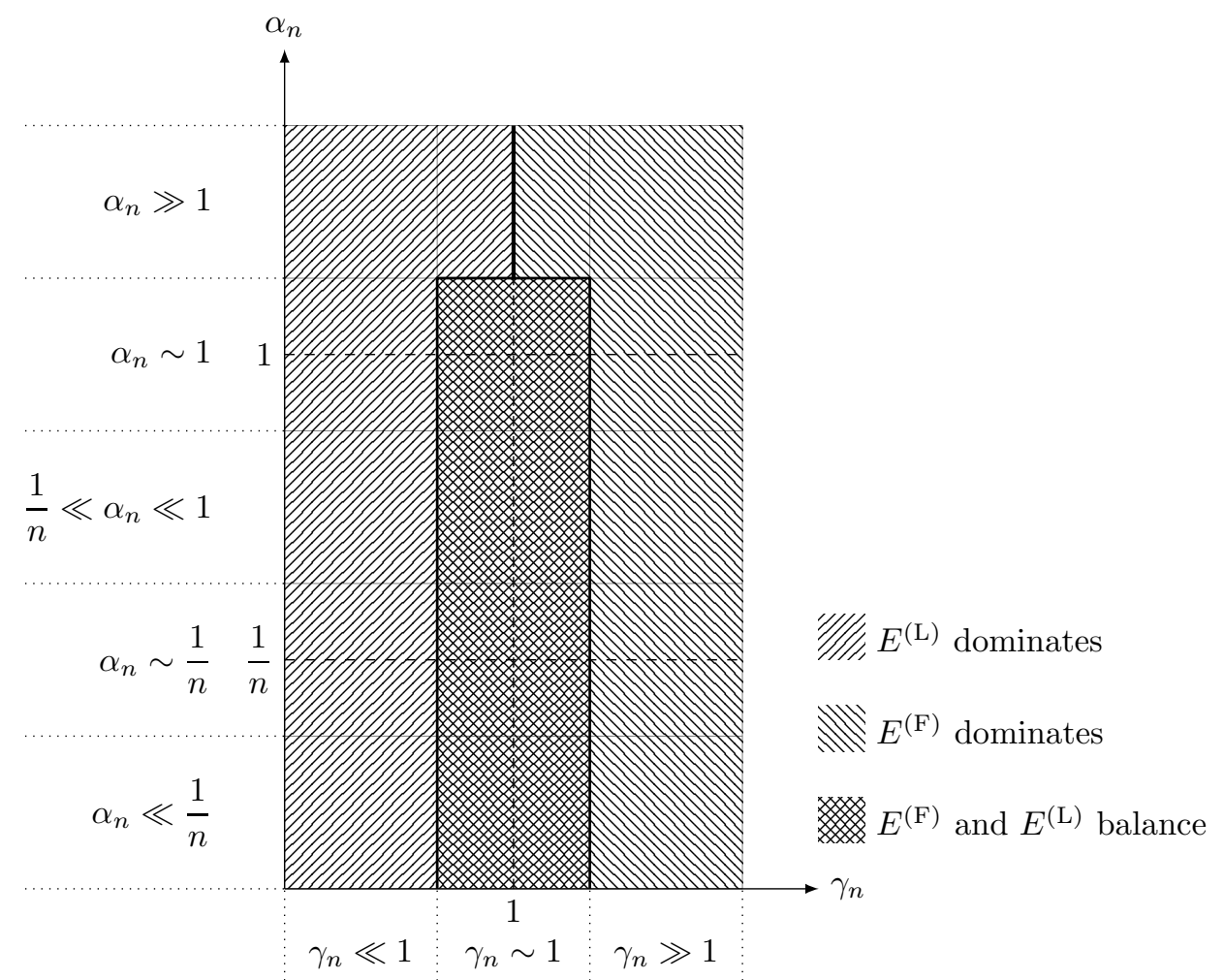

Figure 3. Plot of the regions in parameter space in which either $E^{(\mathrm{L})} \ll E^{(\mathrm{F})}, E^{(\mathrm{F})} \ll E^{(\mathrm{L})}$, or $E^{(\mathrm{F})} \sim E^{(\mathrm{L})}$. The axes show the asymptotic behaviour of $\gamma_{n}$ and $\alpha_{n}$. Although the parameter space is divided in a matrix of five by three blocks, their boundaries do not correspond to specific scalings of $\alpha_{n}$ or $\gamma_{n}$.

\subsection{Discussion}

We started with the question how the finiteness of the domain changes the results from [15. We now discuss the assertions of Theorem 1.1 from this viewpoint, for which we use a schematic plot of the parameter space (Figure 3 ).

- First note that if $\gamma_{n} \gg 1$, i.e. $L_{n} \gg \ell_{n}$, then we recover the same limit energy as in [15. This can be considered a consistency check, showing that the results of this paper generalize [15].

- Moving away from the case of [15], the case $\gamma_{n} \ll 1$ is the simplest: here the finiteness of the domain completely dominates the external forcing (first column in Figure 3). The scaling is independent of the external forcing, and the limit energy is governed by the balance between the interactions and the finiteness of the domain.

- The critical case $\gamma_{n} \rightarrow \gamma$ is more subtle (second column in Figure 3), as can be recognized e.g. in the constant that multiplies the force term of the limit energy. This 
constant is given by

$$
\begin{gathered}
C\left(\gamma ;\left(\alpha_{n}\right)\right):= \begin{cases}\gamma, & \text { if } \alpha_{n} \ll 1 / n, \\
\beta / 2, & \text { if } \alpha_{n} \gg 1, \\
\gamma^{2}, & \text { otherwise, }\end{cases} \\
\text { where } \beta:=\lim _{n \rightarrow \infty} \exp \left(2 \alpha_{n}\left(1-1 / \gamma_{n}\right)\right) .
\end{gathered}
$$

It describes the transition between $E^{(\mathrm{L})} \ll E^{(\mathrm{F})}$ (i.e. $\left.C\left(\gamma ;\left(\alpha_{n}\right)\right)=\infty\right)$, to $E^{(\mathrm{F})} \ll E^{(\mathrm{L})}$ (i.e. $\left.C\left(\gamma ;\left(\alpha_{n}\right)\right)=0\right)$. When $C\left(\gamma ;\left(\alpha_{n}\right)\right) \in(0, \infty)$, both terms of the energy contribute a finite amount. Indeed, for these values of $C\left(\gamma ;\left(\alpha_{n}\right)\right)$ we could have chosen the scaling for $\tilde{\mathbf{x}}$ to be as in (1.7) as well. The $\Gamma$-limit would contain just as much information. However, we use the other scaling (1.9) for purely practical reasons.

When $\alpha_{n} \gg 1$, the transition is very delicate: $C\left(\gamma ;\left(\alpha_{n}\right)\right) \in(0, \infty)$ if $\beta \in(0, \infty)$, which can only occur if $\gamma=1$. This is indicated in Figure 3 by the vertical line at $\gamma_{n} \rightarrow 1$. If $\beta=\infty$, it holds that $E^{(\mathrm{F})}(\mu)=\infty$, and hence the scaling of $\tilde{\mathbf{x}}$ by $L_{n}$ doesn't give a useful limit energy. That is why the case $\beta=\infty$ is excluded in Theorem 1.1. The scaling as given by (1.7) does work. This is made precise by Theorem 4.1 . from which we conclude that $E^{(\mathrm{L})}$ is indeed negligible with respect to $E^{(\mathrm{F})}$ in this case.

- For $\alpha_{n} \gg 1$ and $\gamma_{n}$ bounded such that $\beta \neq \infty$ (i.e. the part of parameter space given by the left half of the first row of Figure 3), the energy $E$ is degenerate in the sense that it is only finite at exactly one point, the measure $\mu=\left.\mathcal{L}\right|_{(0,1)}$. Hence it only contains information about the minimizer. One way to obtain more information in the limit energy is by using a logarithmic rescaling of $E_{n}$. In Theorem 4.4 we state our result that

$$
\frac{1}{2 \alpha_{n}} \log E_{n} \stackrel{\Gamma}{\rightarrow}\left(\mu \mapsto 1-\left\|\frac{d \mu}{d \mathcal{L}}\right\|_{\infty}^{-1}\right) .
$$

- It might be instructive to note that the five expressions for $E^{(\mathrm{i})}(\mu)$ only depend on $\gamma_{n}$ through the choice of rescaling with $\hat{\alpha}_{n}$ versus $\alpha_{n}$. This shows that the presence of the second barrier does not influence the interaction behaviour of the walls.

Summarizing, the finiteness of the domain induces a second length scale - the length of the domain $L_{n}$-in addition to the length scale $\ell_{n}$ generated by the external forcing. We specified three qualitatively different limiting behaviours for the energy, which correspond to the cases $L_{n}$ being asymptotically bigger, smaller, or equal to $\ell_{n}$. This result enables us to test the mutually contradicting dislocation-density models (as mentioned in the introduction) with more freedom in the microscopic setting. As a special case, we are able to test these models when no loading is applied (i.e. $\sigma_{n}=0$ ).

On the other hand, for the parameter regime in which the forcing term is negligible with respect to the effect of the finite domain, it seems unphysical to ignore the effect of negative edge dislocations. One of the reasons that we do not consider a model with negative edge dislocations, is that the effect of nucleation and annihilation of dislocations with opposite sign results in an energy that is not bounded from below. Various methods have been used to circumvent this issue 17, 2, but they each have their drawbacks. We plan to explore the extension of the present results to the case of multiple signs in the future. 
A significant step towards applicability would be to replace the assumption of equispaced slip planes by a stochastic spacing, as also suggested by other authors 20, 34]). If one maintains the wall assumption, then stochastic spacing leads to a different interaction potential $V$, for which no explicit expressions exist, and for which the largedistance behaviour is not yet completely understood 20. However, in the case of stochastically spaced slip planes, dislocations do not form exact walls [28] so that one requires a fully two-dimensional description. A rigorous upscaling in the two-dimensional framework would be the ultimate goal, but that is still far away.

Besides extending the microscopic model to have more freedom in space, one can also consider dislocation dynamics, which is paramount for understanding plasticity. In the case of a linear drag law [22, Ch. 7], these dynamics are described by a gradient flow of the energy. Upscaling the dynamics of the discrete dislocation walls to dislocation densities requires more than just $\Gamma$-convergence of the energies (see e.g. 31]); one also needs lower bounds on the slopes. We plan to return to this question in a future publication.

This paper is organized as follows. We prove Theorem 1.1 in Section 3, which requires a detailed description of our setting and its notation (Section 2.1) followed by crucial arguments that support the proof (Section 2.2 up to and including Section 2.4). This leaves us with the small range of parameters which is excluded in Theorem 1.1, with the question whether the limiting energies still have a unique minimizer (and whether the discrete minimizers converge to it), and with the issue that the limiting energy in the dilute case (i.e. $\alpha_{n} \gg 1$ and $\gamma \leq 1$ ) solely contains information about the minimizer. These three issues are all separately solved in Section 4. In the Appendix we discuss a few technical steps in the proof of Theorem 1.1. and we briefly recall the definition of $\Gamma$-convergence together with its basic properties.

\section{Preliminaries}

\subsection{Notation}

\subsubsection{Basic notation}

- We denote a sequence by $\left(a_{n}\right)$.

- $\chi_{\{A\}}:= \begin{cases}0, & A \text { is true, } \\ \infty, & A \text { is false. }\end{cases}$

- $\overline{\mathbb{R}}:=\mathbb{R} \cup\{ \pm \infty\}$.

- We denote the Lebesgue measure by $\mathcal{L}$.

- For $\xi \in B V(\mathbb{R})$, we denote the distributional derivative by $D \xi$. If $\xi$ is at least weakly differentiable, we use the common notation $\xi^{\prime}$ for the real-valued derivative of $\xi$.

- $\mathcal{P}([0, \infty)):=$ space of probability measures.

- Let $X$ be a metric space and $E: X \rightarrow \overline{\mathbb{R}}$. A subset $Y \subset X$ is said to be energy dense if

$$
\{(y, E(y)) \mid y \in Y\} \subset Y \times \overline{\mathbb{R}} \text { is dense in }\{(x, E(x)) \mid x \in X\},
$$

or equivalently,

$$
\forall x \in X \exists\left(y_{n}\right) \subset Y: y_{n} \rightarrow x \text { and } \lim _{n \rightarrow \infty} E\left(y_{n}\right)=E(x) .
$$


The set $Y$ is said to be lower energy dense in $X$ with respect to $E$ if

$$
\forall x \in X \exists\left(y_{n}\right) \subset Y: y_{n} \rightarrow x \text { and } \limsup _{n \rightarrow \infty} E\left(y_{n}\right) \leq E(x) .
$$

Note that energy density implies lower energy density. We need to prove lower energy density of two sets a number of times, but often it is just as easy to show that they are even energy dense.

- We use the symbols $\sim, \ll$ and $\lesssim$ to precisely denote the different scaling regimes for $\gamma_{n}$ and $\hat{\alpha}_{n}$ or $\alpha_{n}$. They are defined as follows. Let $\left(a_{n}\right),\left(b_{n}\right) \subset \mathbb{R}$, then

$$
\begin{aligned}
& a_{n} \sim b_{n}: \Leftrightarrow \frac{a_{n}}{b_{n}} \text { converges to some } C \in(0, \infty), \\
& a_{n} \ll b_{n}: \Leftrightarrow \limsup _{n \rightarrow \infty} \frac{a_{n}}{b_{n}}=0, \\
& a_{n} \lesssim b_{n}: \Leftrightarrow a_{n} \sim b_{n} \text { or } a_{n} \ll b_{n} .
\end{aligned}
$$

We similarly define $\gg$ and $\gtrsim$. Two sequences $\left(a_{n}\right),\left(b_{n}\right) \subset \mathbb{R}$ do not have to satisfy any of the above criteria. However, these sequences are not important to us, as we shall argue in Remark 2.2 .

In the standard asymptotics literature, $\sim$ typically means $a_{n} / b_{n} \rightarrow 1$. This is expressed here by writing $a_{n}=b_{n}+\mathcal{O}\left(c_{n}\right)$, where a sequence $c_{n} \ll b_{n}$ is specified.

\subsubsection{Difference in notation compared to [15]}

We use a slightly different expression for $K$ and $V$ to simplify formulas. To make the connection clear, we decorate the corresponding quantities in [15] by a sub- or superscript GPPS, in honour of the authors. The connection is given by $K=K_{\mathrm{GPPS}} / \pi^{2}$ and

$$
V(r):=\pi^{2} V_{\mathrm{GPPS}}\left(\frac{r}{\pi}\right) .
$$

Proposition 2.1 (Properties of the interaction potential $V$ ). $V$ as defined by (1.2) satisfies:

(i) $V$ is even;

(ii) $\left.V\right|_{(0, \infty)}$ is strictly convex;

(iii) $V(r)=\log \frac{1}{r}+1-\log 2+\mathcal{O}\left(r^{2}\right)$ for $r \ll 1$;

(iv) $V(r)=2 r e^{-2 r}+\mathcal{O}\left(r e^{-4 r}\right)$ for $r \gg 1$.

\subsubsection{Scaling regimes}

We use the letter $q \in\{1,2,3\}$ to indicate any of the three scaling regimes for $\gamma_{n}$ in Table2 As a result, $q$ labels the columns in Figure 3in decreasing order. We also use $q=0$, which corresponds to $\gamma_{n}=\infty$, to indicate the setting without second barrier (as in [15]). Let us immediately use $q$ to unify the notation for the aspect ratio: let $\alpha_{n}^{(q)}$ be defined by

$$
\alpha_{n}^{(0)}:=\alpha_{n}^{(1)}:=\hat{\alpha}_{n}, \quad \alpha_{n}^{(2)}:=\alpha_{n}^{(3)}:=\alpha_{n} .
$$

Similarly, we introduce $p \in\{1, \ldots, 5\}$ to indicate any of the five scaling regimes for $\hat{\alpha}_{n}$ in Table 1. In decreasing order, $p$ labels the rows in Figure 3. The following list illustrates how we exploit the indices $p$ and $q$ to distinguish scaling regimes: 
- $(p, q)$ : we consider any scaling for $\alpha_{n}^{(q)}$ and $\gamma_{n}$ at the same time.

- $(p, 3)$ : we consider $\gamma_{n} \ll 1$, but no restriction on the scaling of $\alpha_{n}^{(q)}$. We also refer to this by "case $q=3$ ".

- $(5,0): 1 \ll \hat{\alpha}_{n}$ and $\gamma_{n} \ll 1$.

- $(2, q)$ for $q=2,3: \alpha_{n}^{(q)} \sim 1 / n$ and $\gamma_{n} \lesssim 1$.

- $((2-4), q)$ : short-hand notation for $(p, q)$ for $p=2,3,4$. It means that $1 / n \lesssim \alpha_{n}^{(q)} \lesssim 1$ and no restrictions on the scaling of $\gamma_{n}$.

Not all possible sequences $\alpha_{n}^{(q)}$ and $\gamma_{n}$ can be characterized by a single value for $p$ or $q$. Fortunately, the following remark shows that these sequences can never yield a unique limit for the related energy functionals.

Remark 2.2 (Explanation for conditions in Theorem 1.1). Let $\alpha_{n}^{(q)}$ or $\gamma_{n}$ be such that they can not be characterized by a single value for $p$ or $q$. Then there exist at least two subsequences that belong to a different class (or converge to a different constant $\tilde{c}$ or $\gamma$ ). As can be seen from the expression for $E^{(p, q)}(\mu)$, this would give different limit energies, depending along which of these subsequences we take the $\Gamma$-limit, and hence the $\Gamma$-limit does not exist for such sequences $\alpha_{n}^{(q)}$ or $\gamma_{n}$.

\subsubsection{Energies for fixed $n$}

From this point on, we denote the energy as stated in (1.7) and (1.9) by

$$
E_{n}^{(p, q)}=E_{n}^{(p, q ; \mathrm{i})}+E_{n}^{(p, q ; \mathrm{F})}+E_{n}^{(p, q ; \mathrm{L})}: \Omega_{n} \rightarrow \overline{\mathbb{R}},
$$

where

$$
\Omega_{n}:=\left\{\left(x_{1}, \ldots, x_{n}\right) \in[0, \infty)^{n} \mid x_{1} \leq x_{2} \leq \ldots \leq x_{n}\right\} .
$$

Furthermore, we define $x_{0}:=0$ to indicate the pinned dislocation wall at the left barrier.

Now we can explicitly denote all the components of the energies $E_{n}^{(p, q)}$ in terms of the two parameters $\alpha_{n}^{(q)}$ and $\gamma_{n}$ :

$$
\begin{aligned}
E_{n}^{(1, q ; \mathrm{i})}\left(x^{n}\right) & =\frac{1}{n^{2}} \sum_{k=1}^{n} \sum_{j=0}^{n-k} V\left(n \alpha_{n}^{(q)}\left(x_{j+k}^{n}-x_{j}^{n}\right)\right)-\frac{1}{2} \log \frac{e}{2 n \alpha_{n}^{(q)}}, \\
E_{n}^{((2-4), q ; \mathrm{i})}\left(x^{n}\right) & =\frac{\alpha_{n}^{(q)}}{n} \sum_{k=1}^{n} \sum_{j=0}^{n-k} V\left(n \alpha_{n}^{(q)}\left(x_{j+k}^{n}-x_{j}^{n}\right)\right), \\
E_{n}^{(5, q ; \mathrm{i})}\left(x^{n}\right) & =\frac{\exp \left(2\left(\alpha_{n}^{(q)}-1\right)\right)}{n \alpha_{n}^{(q)}} \sum_{k=1}^{n} \sum_{j=0}^{n-k} V\left(n \alpha_{n}^{(q)}\left(x_{j+k}^{n}-x_{j}^{n}\right)\right), \\
E_{n}^{(p,(0-1) ; \mathrm{F})}\left(x^{n}\right) & =\frac{1}{n} \sum_{i=1}^{n} x_{i}^{n}, \\
E_{n}^{(1,(2-3) ; \mathrm{F})}\left(x^{n}\right) & =\gamma_{n} \frac{1}{n} \sum_{i=1}^{n} x_{i}^{n}, \\
E_{n}^{((2-4),(2-3) ; \mathrm{F})}\left(x^{n}\right) & =\gamma_{n}^{2} \frac{1}{n} \sum_{i=1}^{n} x_{i}^{n},
\end{aligned}
$$




$$
\begin{aligned}
& E_{n}^{(5,(2-3) ; \mathrm{F})}\left(x^{n}\right)=\exp \left(2 \alpha_{n}\left(1-\frac{1}{\gamma_{n}}\right)\right) \frac{1}{n} \sum_{i=1}^{n} x_{i}^{n}, \\
& E_{n}^{(p,(0-1) ; \mathrm{L})}\left(x^{n}\right)=\chi_{\left\{x_{n}^{n} \leq \gamma_{n}\right\}}, \\
& E_{n}^{(p,(2-3) ; \mathrm{L})}\left(x^{n}\right)=\chi_{\left\{x_{n}^{n} \leq 1\right\}},
\end{aligned}
$$

The constant in $E_{n}^{(1, q ; i)}\left(x^{n}\right)$ is introduced to balance a constant contribution to the energy for each wall-wall interaction, regardless of their intermediate distance. From the expressions above and $V$ being strictly convex on $(0, \infty)$, it is easy to see that the following proposition holds:

Proposition 2.3 $E_{n}^{(p, q)}: \Omega_{n} \rightarrow \mathbb{R}$ is strictly convex.

\subsubsection{Limit energies}

We continue with the notation for the limit energies. Let

$$
\begin{gathered}
E^{(p, q)}: \mathcal{P}([0, \infty)) \rightarrow \mathbb{R}, \\
E^{(p, q)}(\mu)=E^{(p, q ; \mathrm{i})}(\mu)+E^{(p, q ; \mathrm{F})}(\mu)+E^{(p, q ; \mathrm{L})}(\mu),
\end{gathered}
$$

of which the expressions for the components are listed in Table 1 and Table 2, To be precise, we need to define $\gamma=\infty$ in case $q=0,1$ and $\gamma=0$ in case $q=3$, in order to make sense of the expression of $E^{(5, q ; i)}(\mu)$. Observe that in case $p=5$ and $q=2,3$, the expression for $E^{(p, q)}(\mu)$ reduces to

$$
E^{(5,(2-3))}(\mu)=2 e^{-2} \chi_{\left\{\mu=\left.\mathcal{L}\right|_{[0,1]}\right\}}+\frac{\beta}{2} \mathbb{1}_{\{\gamma=1\}} .
$$

In some cases, it will be useful to reformulate $E^{(p, q)}$ in terms of elements from

$$
X:=\{\xi:(0,1) \rightarrow[0, \infty) \mid \xi \text { non-decreasing }\} .
$$

The elements $\xi \in X$ relate to $\mu \in \mathcal{P}([0, \infty))$ by being the inverse of the cumulative distribution of $\mu$. To state this more precise, we use the following notion of pseudoinvertibility. Let $f:(a, b) \rightarrow(c, d)$ non-decreasing, then we call

$$
f^{-1}(y):=\sup \{x \in(a, b) \mid f(x)<y\}
$$

the pseudo-inverse of $f$. By using the pseudo-inverse, we can denote the relation between $\xi$ and $\mu$ by

$$
\xi=(x \mapsto \mu([0, x]))^{-1}, \quad \mu=D\left(\xi^{-1}\right),
$$

where $D$ stands for the distributional derivative. Later on, in Theorem 2.11 we derive the related metric on $X$, which allows us to prove $\Gamma$-convergence of the energies either on $\mathcal{P}([0, \infty))$ or $X$.

Before writing out explicitly the components of

$$
E^{(p, q)}(\xi)=E^{(p, q ; \mathrm{i})}(\xi)+E^{(p, q ; \mathrm{F})}(\xi)+E^{(p, q ; \mathrm{L})}(\xi),
$$

for $\xi \in X$, we note that the following equalities follow from (2.4)

$$
\max \operatorname{supp} \mu=\sup \xi, \quad\|\rho\|_{\infty}=\frac{1}{\inf \xi^{\prime}},
$$


where the second equality only makes sense if $\xi \in W_{\text {incr }}^{1,1}$ and if $\mu$ is absolutely continuous with $\rho=d \mu / d \mathcal{L}$. Together with these inequalities, it is easy to see

$$
\begin{aligned}
E^{(3,(0-3) ; \mathrm{i})}(\xi) & :=\left(\int_{0}^{\infty} V\right) \int_{0}^{1} \frac{1}{\xi^{\prime}} \\
E^{(4,(0-3) ; \mathrm{i})}(\xi) & :=\tilde{c} \int_{0}^{1}\left(\sum_{k=1}^{\infty} V\left(\tilde{c} k \xi^{\prime}\right)\right) \\
E^{(5,(0-3) ; \mathrm{i})}(\xi) & := \begin{cases}2 e^{-2} \mathbb{1}_{\{\gamma \leq 1\}}, & \text { if } \xi^{\prime} \geq 1 \text { a.e. } \\
\infty, & \text { otherwise, }\end{cases} \\
E^{((1-5),(0-1) ; \mathrm{F})}(\xi) & :=\int_{0}^{1} \xi, \\
E^{((1-5), 2 ; \mathrm{F})}(\xi) & :=C^{(p)}(\gamma) \int_{0}^{1} \xi, \\
E^{((1-5), 3 ; \mathrm{F})}(\xi) & :=0, \\
E^{((1-5),(0-1) ; \mathrm{L})}(\xi) & :=0, \\
E^{((1-5),(2-3) ; \mathrm{L})}(\xi) & :=\chi_{\{\sup \xi \leq 1\}},
\end{aligned}
$$

where $C^{(p)}(\gamma)$ is the same constant as defined in (1.11) (we have changed the second argument to $p$ for convenience).

In cases $p=3,4,5$, it turns out to be convenient to use both descriptions of $E^{(p, q)}$. Since it will be clear from the context in this paper which of the two descriptions we use, we do not make a distinction notation-wise.

Just as in (1.10) we can regard $E_{n}^{(p, q)}$ as

$$
\begin{aligned}
& E_{n}^{(p, q)}: X \rightarrow \overline{\mathbb{R}} \\
& E_{n}^{(p, q)}(\xi):= \begin{cases}E_{n}^{(p, q)}(\xi), & \text { if } \exists x \in \Omega_{n}: \xi=\xi_{n} \text { in the sense of Definition 2.9 } \\
\infty, & \text { otherwise. }\end{cases}
\end{aligned}
$$

Again, we will not make a notational distinction.

\subsection{Using density to construct recovery sequences}

Lemma 2.4 will serve as the backbone for the proof of Theorem 1.1 .

Lemma 2.4 (limsup inequality for a dense subset). Let $M$ be a metric space, $M_{1} \subset M$ dense, $F_{n}, F: M \rightarrow \mathbb{R}$. If

(i) $\forall v \in M_{1} \exists\left(u_{n}\right) \subset M: u_{n} \rightarrow v$ and $\limsup _{n \rightarrow \infty} F_{n}\left(u_{n}\right) \leq F(v)$, and

(ii) $\forall u \in M \exists\left(v_{n}\right) \subset M_{1}: v_{n} \rightarrow u$ and $\limsup _{n \rightarrow \infty} F\left(v_{n}\right) \leq F(u)$,

then $\forall u \in M \exists\left(u_{n}\right) \subset M: u_{n} \rightarrow u$ and $\limsup _{n \rightarrow \infty}^{n \rightarrow \infty} F_{n}\left(u_{n}\right) \leq F(u)$.

Remark 2.5 The proof of Lemma 2.4 is based on a diagonal argument. See e.g. 8 , Proposition 6.2] for the proof of a similar statement. Minor, obvious adjustments to that proof are needed to prove Lemma 2.4 
The following Lemma turns out to be very useful in our application of Lemma 2.4. It gives a sufficient condition for condition $(i i)$, which consists of easier subproblems. In a way, it shows that one can show condition (ii) iteratively. Since the proof can be done by a straight-forward diagonal argument, we do not show it here.

Lemma 2.6 (Alternative for Lemma 2.4, condition (ii)). Let $M$ be a metric space, $M_{1} \subset$ $M$ dense, $k \in\{2,3, \ldots\}, M_{1} \subset M_{2} \subset \ldots M_{k}:=M$, and $F_{n}, F: M \rightarrow \mathbb{R}$. If

$$
\forall j \in\{1, \ldots, k-1\} \forall u \in M_{j+1} \exists\left(v_{n}\right) \subset M_{j}: v_{n} \rightarrow u \text { and } \limsup _{n \rightarrow \infty} E\left(v_{n}\right) \leq E(u),
$$

then condition (ii) of Lemma 2.4 is satisfied.

\subsection{Link between $\mathcal{P}([0, \infty))$ and $X$}

In (2.4) we have shown how elements from $\mathcal{P}([0, \infty))$ relate to those of $X$. Here, we like to give a topology on $X$ for which $\Gamma$-convergence of $E_{n}^{(p, q)}$ to $E^{(p, q)}$ on $X$ with respect to that topology is equivalent to $\Gamma$-convergence of $E_{n}^{(p, q)}$ to $E^{(p, q)}$ on $\mathcal{P}([0, \infty))$ with respect to narrow convergence. This statement follows easily from Theorem 2.11 Before stating it, we need two definitions:

Definition 2.7 (Topology on $X$ ). Let $\xi_{n}, \xi \in X$. We say that $\xi_{n} \rightarrow \xi$ in $B V_{l o c}(0,1)$ if for all $\delta \in(0,1)$ we have that $\xi_{n} \rightarrow \xi$ in $L^{1}(0,1-\delta)$ and $D \xi_{n} \rightarrow D \xi$ in $\mathcal{P}((0,1-\delta))$ with respect to the narrow topology, where $D$ is the distributional derivative.

Remark 2.8 Our motivation for using $\delta \in(0,1)$ instead of just taking $\delta=0$, is that $\xi(s)$ may go to $\infty$ if $s \uparrow 1$. This happens when the related $\mu \in \mathcal{P}([0, \infty))$ has unbounded support.

Definition 2.9 (Embedding discrete wall density). For a sequence of $(n+1)$-tuples denoted by $\left(\left(x_{i}^{n}\right)_{i=0}^{n}\right)_{n \in \mathbb{N}_{+}}$that satisfies $x_{0}^{n}=0$ and $x_{i-1}^{n} \leq x_{i}^{n}$ for all $n \in \mathbb{N}_{+}$and for all $i \in\{1, \ldots, n\}$, we define $\left(\mu_{n}\right) \subset \mathcal{P}([0, \infty))$ and $\left(\xi_{n}\right) \subset W^{1, \infty}(0,1)$ by

$$
\begin{gathered}
\mu_{n}:=\frac{1}{n} \sum_{i=1}^{n} \delta_{x_{i}^{n}}, \\
\xi_{n}(s):=x_{i-1}^{n}+n\left(x_{i}^{n}-x_{i-1}^{n}\right)\left(s-\frac{i-1}{n}\right), \quad \text { for } s \in\left(\frac{i-1}{n}, \frac{i}{n}\right) .
\end{gathered}
$$

Remark 2.10 We have made the choice to exclude $x_{0}^{n}$ from the definition of $\mu_{n}$.

A useful interpretation of $\mu_{n}$ and $\xi_{n}$ is as follows. For a Borel set $A$ of $X$, the fraction of dislocation walls in $A$ is given by $\mu_{n}(A) . \xi_{n}$ uses the property that the walls are ordered by their position. $\xi_{n}(i / n)$ is the position of wall $i$. All the intermediate values of $\xi_{n}$ are chosen to be convenient in the $\Gamma$-convergence proofs.

Theorem 2.11 (Link between $\mu$ and $\xi[15])$. Let $\left(\left(x_{i}^{n}\right)_{i=0}^{n}\right)_{n \in \mathbb{N}_{+}},\left(\mu_{n}\right),\left(\xi_{n}\right)$ as in Definition 2.9. Then the following two statements are equivalent:

(i) $\xi_{n} \rightarrow \xi$ in $B V_{l o c}(0,1)$, 
(ii) $\mu_{n} \rightarrow \mu$.

Moreover, if any of the two statements above hold, and $\xi^{-1} \in W^{1,1}(0, \infty)$, then

$$
\rho:=\frac{d \mu}{d \mathcal{L}}=\left(\xi^{-1}\right)^{\prime}
$$

\subsection{Properties of the "F" and "L" part of the energies}

The energies $E_{n}^{(p, q ; \mathrm{F})}$ and $E_{n}^{(p, q ; \mathrm{L})}$ have special structure. The related properties are useful in reducing the complexity of the proof of Theorem 1.1. Here, we make these properties precise.

Let

$$
C_{n}^{(p)}\left(\gamma_{n}, \alpha_{n}\right):= \begin{cases}\gamma_{n}, & \text { if } p=1, \\ \gamma_{n}^{2}, & \text { if } p \in\{2,3,4\}, \\ \exp \left(2 \alpha_{n}\left(1-1 / \gamma_{n}\right)\right), & \text { if } p=5 .\end{cases}
$$

If $q=3$, we have $C_{n}^{(p)}\left(\gamma_{n}, \alpha_{n}\right) \rightarrow 0$. If $q=2$, we obtain $C_{n}^{(p)}\left(\gamma_{n}, \alpha_{n}\right) \rightarrow C^{(p)}(\gamma)$. We will require $C^{(p)}(\gamma)$ to be finite. This means that for $p=1$, we have to impose $\beta<\infty$. Note that this is exactly what we require in Theorem 1.1.

Without violating (1.10), we can regard $E_{n}^{(p, q ; \mathrm{F})}, E_{n}^{(p, q ; \mathrm{L})}: \mathcal{P}([0, \infty)) \rightarrow \mathbb{R}$ as

$$
\begin{aligned}
& E_{n}^{(p,(0-1) ; \mathrm{F})}(\mu):=E^{(p,(0-1) ; \mathrm{F})}(\mu)=\int_{0}^{\infty} x d \mu(x) \\
& E_{n}^{(p,(2-3) ; \mathrm{F})}(\mu):=C_{n}^{(p)}\left(\gamma_{n}, \alpha_{n}\right) \int_{0}^{\infty} x d \mu(x) \\
& E_{n}^{(p,(2-3) ; \mathrm{L})}(\mu):=E^{(p,(2-3) ; \mathrm{L})}(\mu)
\end{aligned}
$$

The following proposition is now a straightforward consequence of the statements above:

Proposition 2.12 (Continuous convergence of the force term). For any $p \in\{1, \ldots, 5\}$ and any $q \in\{0, \ldots, 3\}$ (except for the case $p=5, q=2$ and $\beta=\infty$ (see (1.12) )),

$$
E_{n}^{(p, q ; \mathrm{F})} \rightarrow E^{(p, q ; \mathrm{F})},
$$

where the convergence is as in Definition B.2 (i.e. continuous convergence) on the space $\mathcal{P}([0,1])$ with respect to the narrow topology.

Remark 2.13 Proposition 2.12 basically allows us to decouple the force term from the energy in the proof of Theorem 1.1 whenever $q=2,3$. This is mainly due to Theorem B.3. but we need additional arguments because the energy is defined on $\mathcal{P}([0, \infty))$ instead of $\mathcal{P}([0,1])$.

Proposition 2.12 does not always apply due to the restriction to $\mathcal{P}([0,1])$. In that case, we still have lower semicontinuity, which also holds for $E^{(p,(2-3) ; \mathrm{L})}$ :

Proposition $2.14 E^{(p,(0-1) ; \mathrm{F})}$ and $E^{(p,(2-3) ; \mathrm{L})}$ are lower semicontinuous on $\mathcal{P}([0, \infty))$ with respect to the narrow topology. 
Proof of Proposition 2.14 Both $E^{(p,(0-1) ; \mathrm{F})}$ and $E^{(p,(2-3) ; \mathrm{L})}$ can be written as integrals over lower semicontinuous functions that are bounded from below. Lower semicontinuity of $E^{(p,(0-1) ; \mathrm{F})}$ and $E^{(p,(2-3) ; \mathrm{L})}$ follows from the Portmanteau Theorem.

\section{Proof of Theorem 1.1}

Theorem 1.1 consists of two statements; a compactness property and $\Gamma$-convergence of the energies. The first can be proved in a few lines, which we do next. After that, we continue with the proof of the $\Gamma$-convergence.

By the compactness property, we mean that if for some $\left(\mu_{n}\right) \subset \mathcal{P}([0, \infty))$ it holds that $E_{n}^{(p, q)}\left(\mu_{n}\right)$ is bounded, then $\left(\mu_{n}\right)$ is compact in the narrow topology. For $q=0$ this is given by [15, Theorem 1]. For $q=1$, we have $E_{n}^{(p, 1)} \geq E_{n}^{(p, 0)}$, so the compactness property follows easily form the case $q=0$. For $q=2,3$, we have $E_{n}^{(p,(2-3))}\left(\mu_{n}\right) \leq C$ implies $E_{n}^{(p,(2-3) ; \mathrm{L})}\left(\mu_{n}\right)=0$, and hence supp $\mu_{n} \subset[0,1]$. This gives tightness of $\left(\mu_{n}\right)$, and by e.g. Prokhorov's Theorem the compactness property follows.

We prove $\Gamma$-convergence of the energies by establishing the two inequalities

$$
\text { for all } \mu_{n} \rightarrow \mu, \quad \liminf _{n \rightarrow \infty} E_{n}^{(p, q)}\left(\mu_{n}\right) \geq E^{(p, q)}(\mu),
$$

for all $\mu$ there exists $\mu_{n} \rightarrow \mu$ such that $\limsup _{n \rightarrow \infty} E_{n}^{(p, q)}\left(\mu_{n}\right) \leq E^{(p, q)}(\mu)$,

for all $p=1, \ldots, 5$ and $q=0, \ldots, 3$ (except for the case $(p, q)=(5,2)$ and $\beta=\infty$ ). Here $\mu_{n}$ and $\mu$ are probability measures on $[0, \infty)$. Note that it is sufficient to prove (3.1b) for all $\mu$ with $E^{(p, q)}(\mu)<\infty$.

In these inequalities, $E_{n}^{(p, q)}$ and $E^{(p, q)}$ are sums of three terms

$$
E_{n}^{(p, q)}=E_{n}^{(p, q ; \mathrm{i})}+E_{n}^{(p, q ; \mathrm{F})}+E_{n}^{(p, q ; \mathrm{L})}, \quad \text { and } \quad E^{(p, q)}=E^{(p, q ; \mathrm{i})}+E^{(p, q ; \mathrm{F})}+E^{(p, q ; \mathrm{L})},
$$

which are given in the list starting on page 11 and in Tables 1 and 2. Since similar results were proved in [15] for similar energies without the final ("L") term, we will be using many results from [15. The following lemma lists them. It uses the following (sub)spaces:

$$
\begin{aligned}
\mathcal{Y} & :=\left\{\mu \in \mathcal{P}([0, \infty)) \mid \text { supp } \mu \text { bounded, } \mu \ll \mathcal{L}, \text { and } \frac{d \mu}{d \mathcal{L}} \in L^{\infty}(0, \infty)\right\} \\
X & =\{\xi:(0,1) \rightarrow[0, \infty) \mid \xi \text { non-decreasing }\} \\
Y^{(4)} & :=W_{\text {incr }}^{1,1}(0,1) \\
Y^{(3)} & :=\left\{\xi \in Y^{(4)} \mid \xi^{\prime} \geq \varepsilon \text { for some } \varepsilon>0\right\} \\
\tilde{Y}^{(5)} & :=\left\{\xi \in Y^{(4)} \mid \xi \text { piece-wise affine }\right\} .
\end{aligned}
$$

The tilde on $\tilde{Y}^{(5)}$ is due to us using another definition for $Y^{(5)}$ in the proof of Theorem 1.1

Lemma 3.1 (Results from [15])

(i) (liminf inequality). Let $\mu_{n}, \mu \in \mathcal{P}([0, \infty))$, and $\mu_{n} \rightarrow \mu$. For all $p \in\{1, \ldots, 5\}$ and all $q \in\{0, \ldots, 3\}$, we have

$$
\liminf _{n \rightarrow \infty} E_{n}^{(p, q ; \mathrm{i})}\left(\mu_{n}\right) \geq E^{(p, q ; \mathrm{i})}(\mu) .
$$


In addition, for all $0 \leq I<J \leq n$,

$$
\frac{1}{n} \sum_{k=1}^{n} \sum_{j=0}^{n-k} V\left(n \alpha_{n}\left(x_{j+k}-x_{j}\right)\right) \geq \frac{1}{n}(J-I) V\left(n \alpha_{n} \frac{x_{J}-x_{I}}{J-I}\right) .
$$

(ii) (limsup inequality). Let $p \in\{1, \ldots, 5\}, q \in\{0,1\}, \mu \in \mathcal{P}([0, \infty)$ ). Then there exists $\mu_{n} \rightarrow \mu$ such that

$$
\limsup _{n \rightarrow \infty} E_{n}^{(p, q)}\left(\mu_{n}\right) \leq E^{(p, q)}(\mu) .
$$

(iii) (Condition (1i) of Lemma 2.4). Fix $p \in\{1, \ldots, 5\}$. If $p \leq 2$, let $\mu \in \mathcal{Y}$. If $p=3$, 4 , let $\xi \in Y^{(p)} ;$ if $p=5$, let $\xi \in \tilde{Y}^{(5)}$. Let

$$
\begin{aligned}
x_{i}^{(p), n} & :=\inf \{x \in[0, \infty) \mid \mu([0, x]) \geq i / n\}, \quad \text { for } p=1,2, \\
x_{i}^{(p), n} & :=\xi\left(\frac{i}{n}\right), \quad \text { for } p=3,4, \\
x_{i}^{(p), n} & :=\left(1+\varepsilon_{n}\right) \xi\left(\frac{i}{n}\right), \quad \text { for } p=5,
\end{aligned}
$$

for some sequence $\varepsilon_{n} \downarrow 0$. Let $\left(\mu_{n}^{(p)}\right)$ be defined as in (2.5al). Then

$$
\begin{gathered}
\limsup _{n \rightarrow \infty} E_{n}^{(p, 0)}\left(\mu_{n}^{(p)}\right) \leq E^{(p, 0)}\left(\mu^{(p)}\right), \\
\limsup _{n \rightarrow \infty} E_{n}^{(p, 0 ; \mathrm{i})}\left(\mu_{n}^{(p)}\right) \leq E^{(p, 0 ; \mathrm{i})}\left(\mu^{(p)}\right),
\end{gathered}
$$

where $\mu^{(p)}:=\mu$ if $p \leq 2$, and $\mu^{(p)}:=\left(\xi^{-1}\right)^{\prime}$ (as in (2.4) ) else.

(iv) (Condition (iii) of Lemma 2.4). Fix $p \in\{1, \ldots, 5\}$. If $p \leq 2$, let $M:=\mathcal{P}([0, \infty))$ and $M_{1}:=\mathcal{Y}$, otherwise let $M:=X$ and $M_{1}:=Y^{(p)}$. Then condition (iii) of Lemma 2.4 holds for $F:=E^{(p, 0)}$.

We now continue with the two inequalities (3.1).

\subsection{The liminf inequality $3.1 a$ )}

In cases $q=0,1$ either the domain is $[0, \infty)(q=0)$ or after rescaling the right-hand bound converges to $+\infty(q=1)$. Therefore the domain restriction enforced by $E_{n}^{(p, q ; \mathrm{L})}$ becomes unimportant in the limit $n \rightarrow \infty$, and for all $p$ we can simply disregard it:

$$
\begin{aligned}
\liminf _{n \rightarrow \infty} E_{n}^{(p, q)}\left(\mu_{n}\right) \stackrel{\text { [.2), (2.8) }}{\geq} E^{(p, q ; \mathrm{i})}(\mu)+E^{(p, q ; \mathrm{F})}(\mu)+\liminf _{n \rightarrow \infty} E_{n}^{(p, q ; \mathrm{L})}(\mu) \\
\geq E^{(p, q ; \mathrm{i})}(\mu)+E^{(p, q ; \mathrm{F})}(\mu)=E^{(p, q)}(\mu),
\end{aligned}
$$

which proves (3.1a) for all $p$ and for $q=0,1$.

In cases $q=2,3$, where the rescaled domain is [0,1], the functional $E_{n}^{(p, q ; \mathrm{L})}$ becomes important. When $q=2,3, E_{n}^{(p, q ; \mathrm{L})}$ is independent of $n$ (see $(2.7 \mathrm{c})$ ) and lower semicontinuous with respect to the narrow convergence (see Proposition 2.14). We then calculate for $p \in\{1, \ldots, 4\}$ and $q=2,3$,

$$
\liminf _{n \rightarrow \infty} E_{n}^{(p, q)}\left(\mu_{n}\right) \stackrel{(3.2),(2.8)}{\geq} E^{(p, q ; \mathrm{i})}(\mu)+E^{(p, q ; \mathrm{F})}(\mu)+\liminf _{n \rightarrow \infty} E^{(p, q ; \mathrm{L})}\left(\mu_{n}\right)
$$




$$
=E^{(p, q ; \mathrm{i})}(\mu)+E^{(p, q ; \mathrm{F})}(\mu)+E^{(p, q ; \mathrm{L})}(\mu)=E^{(p, q)}(\mu) .
$$

This proves (3.1a ) for these cases.

Finally, we discuss the case $q=2,3$ and $p=5$. Here the boundedness of the domain and the exponential behaviour of the tails of $V$ create a behaviour that is different from that on unbounded domains. We calculate, for any $0 \leq I<J \leq n$,

$$
\begin{aligned}
E_{n}^{(5, q ; \mathrm{i})}\left(\mu_{n}\right) & =\frac{\exp \left(2\left(\alpha_{n}-1\right)\right)}{n \alpha_{n}} \sum_{k=1}^{n} \sum_{j=0}^{n-k} V\left(n \alpha_{n}\left(x_{j+k}^{n}-x_{j}^{n}\right)\right) \\
& \stackrel{\text { (3.3) }}{\geq} \frac{\exp \left(2\left(\alpha_{n}-1\right)\right)}{\alpha_{n}} \frac{1}{n}(J-I) V\left(n \alpha_{n} \frac{x_{J}^{n}-x_{I}^{n}}{J-I}\right) .
\end{aligned}
$$

Taking $I=0$ and $J=n$ in this expression, we find that

$$
\begin{aligned}
E_{n}^{(5, q ; \mathrm{i})}\left(\mu_{n}\right) & \geq \frac{\exp \left(2\left(\alpha_{n}-1\right)\right)}{\alpha_{n}} V\left(\alpha_{n}\left(x_{n}^{n}-x_{0}^{n}\right)\right) & & \\
& \geq \frac{\exp \left(2\left(\alpha_{n}-1\right)\right)}{\alpha_{n}} V\left(\alpha_{n}\right) & & \text { since } x_{n}^{n} \leq 1 \\
& =2 e^{-2}+\mathcal{O}\left(e^{-2 \alpha_{n}}\right) & & \text { by Prop. 2.1(iv). }
\end{aligned}
$$

Therefore

$$
\liminf _{n \rightarrow \infty} E_{n}^{(5, q)}\left(\mu_{n}\right) \geq 2 e^{-2}+\liminf _{n \rightarrow \infty}\left[E_{n}^{(5, q ; \mathrm{F})}\left(\mu_{n}\right)+E_{n}^{(5, q ; \mathrm{L})}\left(\mu_{n}\right)\right] \geq 2 e^{-2} .
$$

In order to show that $\lim _{\inf _{n \rightarrow \infty}} E_{n}^{(5, q)}\left(\mu_{n}\right) \geq E^{(5, q)}(\mu)$, we still need to show that $\liminf _{n \rightarrow \infty} E_{n}^{(5, q)}\left(\mu_{n}\right)=\infty$ whenever $\mu \neq\left.\mathcal{L}\right|_{[0,1]}$. If $\operatorname{supp} \mu \nsubseteq[0,1]$, we have that $E_{n}^{(5, q ; \mathrm{L})}\left(\mu_{n}\right)=\infty$ by (2.7c) and Proposition 2.14. If $\operatorname{supp} \mu \subset[0,1]$ and $\mu \neq\left.\mathcal{L}\right|_{[0,1]}$, there exists an interval $(a, b) \subset \mathbb{R}$ such that $\delta:=(b-a)^{-1} \mu((a, b))>1$. Define $I_{n}$ and $J_{n}$ by

$$
x_{I_{n}}^{n}=\min _{i}\left\{x_{i}^{n} \mid x_{i}^{n}>a\right\} \quad \text { and } \quad x_{J_{n}}^{n}=\max _{i}\left\{x_{i}^{n} \mid x_{i}^{n}<b\right\} .
$$

Using Prokhorov's characterization of narrow convergence, we calculate

$\limsup _{n \rightarrow \infty} \delta\left(x_{J_{n}}^{n}-x_{I_{n}}^{n}\right) \leq \delta(b-a)=\mu((a, b)) \leq \liminf _{n \rightarrow \infty} \mu_{n}((a, b))=\liminf _{n \rightarrow \infty} \frac{1}{n}\left(J_{n}-I_{n}+1\right)$,

and therefore

$$
\limsup _{n \rightarrow \infty} n \frac{x_{J_{n}}^{n}-x_{I_{n}}^{n}}{J_{n}-I_{n}} \leq \frac{1}{\delta}
$$

Continuing from (3.10) we then find

$$
\begin{aligned}
E_{n}^{(5, q ; \mathrm{i})}\left(\mu_{n}\right) & \geq \frac{\exp \left(2\left(\alpha_{n}-1\right)\right)}{\alpha_{n}} \frac{1}{n}\left(J_{n}-I_{n}\right) V\left(\alpha_{n} \delta^{-1}\right) \\
& \geq 2 e^{-2}(b-a) \exp \left[2 \alpha_{n}\left(1-\delta^{-1}\right)\right]\left(1+\mathcal{O}\left(e^{-2 \alpha_{n} / \delta}\right)\right) .
\end{aligned}
$$

This converges to $+\infty$ since $\delta>1$. 


\subsection{The limsup inequality 3.16}

The case $q=0$. When $q=0,(\underline{3.1 b})$ is given by Lemma 3.1)(ii). However, for the specific case $p=5$, we present an alternative proof here. The proof is easier and more explicit than the proof as given in [15. Moreover, the arguments in the following proof are easier to extend to the cases in which $q \neq 0$.

We conclude (3.1b) from Lemma 2.4 after showing that its two conditions are satisfied. We use Lemma 2.4 with the subset

$$
Y^{(5)}:=\left\{\xi \in Y_{1}^{(4)} \mid \inf \xi^{\prime}>1\right\} .
$$

Condition (ii). Let $\xi \in Y^{(5)}$. We construct $\xi_{n}$ by using linear interpolation (see (2.5b) ) with $x_{i}^{n}:=\xi(i / n)$. Observe that for any $i, j \in\{0, \ldots, n\}$ with $i>j$, we have the estimate

$$
\left(x_{i}^{n}-x_{j}^{n}\right)=|\xi(i / n)-\xi(j / n)|=\int_{j / n}^{i / n} \xi^{\prime} \geq\left(\inf \xi^{\prime}\right) \frac{i-j}{n} .
$$

Let $m:=\inf \xi^{\prime}>1$. We calculate

$$
\begin{aligned}
& \sum_{k=1}^{n} \sum_{j=0}^{n-k} V\left(n \alpha_{n}\left(x_{j+k}^{n}-x_{j}^{n}\right)\right) \\
& \leq \sum_{k=1}^{n} \sum_{j=0}^{n-k} V\left(n \alpha_{n} m \frac{k}{n}\right) \quad \text { by (3.13) and } V \text { decreasing } \\
& =\sum_{k=1}^{n}(n-k+1) 2 m k \alpha_{n} e^{-2 m k \alpha_{n}}\left(1+\mathcal{O}\left(e^{-2 m k \alpha_{n}}\right)\right) \quad \text { by Prop. 2.1(iv) } \\
& \leq 2 m n \alpha_{n}\left(1+\mathcal{O}\left(e^{-2 m \alpha_{n}}\right)\right) \sum_{k=1}^{n} k e^{-2 m k \alpha_{n}} \\
& =2 m n \alpha_{n}\left(1+\mathcal{O}\left(e^{-2 m \alpha_{n}}\right)\right) e^{-2 m \alpha_{n}}\left(1+\mathcal{O}\left(e^{-2 m \alpha_{n}}\right)\right),
\end{aligned}
$$

from which it follows that

$$
\begin{aligned}
E_{n}^{(5,0 ; \mathrm{i})}\left(\xi_{n}\right) & =\frac{\exp \left(2\left(\alpha_{n}-1\right)\right)}{n \alpha_{n}} \sum_{k=1}^{n} \sum_{j=0}^{n-k} V\left(n \alpha_{n}\left(x_{j+k}^{n}-x_{j}^{n}\right)\right) \\
& \leq \frac{2 m}{e^{2}} e^{-2 \alpha_{n}(m-1)}\left(1+\mathcal{O}\left(e^{-2 m \alpha_{n}}\right)\right) \rightarrow 0 .
\end{aligned}
$$

It remains to show that the limsup also holds for the force term. As $\xi(1)<\infty$, it is allowed to use Proposition 2.12 to conclude that $E_{n}^{(5,0 ; \mathrm{F})} \rightarrow E^{(5,0 ; \mathrm{F})}$ continuously.

Condition (iii). By Lemma 2.6 it is enough to show that the following two inclusions are energy dense:

$$
Y^{(5)} \subset Y^{(4)} \subset X \quad \text { with respect to } E^{(5,0)} \text {. }
$$

Energy density of the second inclusion follows from Theorem A.2. The first inequality is easy to prove: take $\xi \in Y^{(4)}$ with $E^{(5,0)}(\xi)<\infty$. This implies inf $\xi^{\prime} \geq 1$. Hence $\xi_{n}:=$ $(t \mapsto \xi(t)+t / n) \in Y^{(5)}, E^{(5,0 ; \mathrm{i})}\left(\xi_{n}\right)=0=E^{(5,0 ; \mathrm{i})}(\xi)$, and $E^{(5,0 ; \mathrm{F})}\left(\xi_{n}\right) \rightarrow E^{(5,0 ; \mathrm{F})}(\xi)$. This completes the proof for case $(p, q)=(5,0)$.

Case $q=1$. We continue with case $q=1$ for any $p$. The expressions for $E_{n}^{(p, 1)}$ 
and $E^{(p, 1)}$ are very similar to those from case $q=0$, because both the interaction and force term of the related energies are the same. However, the presence of the second barrier may make the recovery sequence as given implicitly by Lemma 3.1(iii) not applicable. Our strategy to solve this issue is to take the explicitly given recovery sequence (only for special choices for $\mu$ (see (3.5) - (3.7)) ), show that these recovery sequences also work in case $q=1$, and extrapolate these results to general $\mu \in \mathcal{P}([0, \infty))$ via Lemma 2.4.

If $p \leq 2$, let $\mu \in \mathcal{Y}$, otherwise let $\xi \in Y^{(p)}$ and define $\mu:=\left(\xi^{-1}\right)^{\prime}$ (as in (2.4)). Let $\mu_{n}$ as in Lemma 3.1(iii). By using this Lemma and max supp $\mu_{n} \leq C$, we obtain

$$
\begin{aligned}
\limsup _{n \rightarrow \infty} E_{n}^{(p, 1)}\left(\mu_{n}\right) & \leq \limsup _{n \rightarrow \infty} E_{n}^{(p, 0)}\left(\mu_{n}\right)+\limsup _{n \rightarrow \infty} E_{n}^{(p, 1 ; \mathrm{L})}\left(\mu_{n}\right) \\
& \leq E^{(p, 0)}(\mu)=E^{(p, 1)}(\mu) .
\end{aligned}
$$

Together with Lemma 3.1(iv) and the observation that $E^{(p, 1)}=E^{(p, 0)}$, we see that the two conditions from Lemma 2.4 are satisfied, from which (3.1b) follows.

Cases $q=2,3$. Here we separate the proof for $p \leq 4$ and $p=5$. In the latter case, we have that $E^{(5, q)}(\mu)$ can only be finite if $\mu=\left.\mathcal{L}\right|_{[0,1]}$, for which the proof requires a different argument.

We start with $p \leq 4$. Note that the energies are much alike for $q=2,3$ : we have $E_{n}^{(p, 2)}=E_{n}^{(p, 3)}$ and $E^{(p, 2)}=E^{(p, 3)}+E^{(p, 2 ; \mathrm{F})}$. Hence we take $q \in\{2,3\}$ arbitrary.

Since we can restrict ourselves to those $\mu \in \mathcal{P}([0, \infty))$ for which $E^{(p, q ; \mathrm{L})}(\mu)$ is finite, we can assume that $\operatorname{supp} \mu \subset[0,1]$ and $E^{(p, q ; \mathrm{L})}(\mu)=0$. We prove (3.1b) by applying Lemma 2.4 to the following spaces:

$$
\begin{aligned}
\mathcal{X}_{1} & :=\{\mu \in \mathcal{P}([0, \infty)) \mid \operatorname{supp} \mu \subset[0,1]\}, \\
\mathcal{Y}_{1} & :=\{\mu \in \mathcal{Y} \mid \operatorname{supp} \mu \subset[0,1]\}, \\
X_{1} & :=\{\xi \in X \mid \sup \xi \leq 1\}, \\
Y_{1}^{(p)} & :=\left\{\xi \in Y^{(p)} \mid \sup \xi \leq 1\right\}, \quad \text { for } p=3,4 .
\end{aligned}
$$

It remains to show that the two conditions of Lemma 2.4 are satisfied:

Condition (ii). Let $p=1,2$ and $\mu \in \mathcal{Y}_{1}$. Let $\mu_{n}$ as in Lemma 3.1(iii). Observe that $\operatorname{supp} \mu_{n} \subset[0,1]$, so

$$
\begin{aligned}
& \limsup _{n \rightarrow \infty} E_{n}^{(p, q)}\left(\mu_{n}\right) \\
& \leq \quad \limsup _{n \rightarrow \infty} E_{n}^{(p, q ; \mathrm{i})}\left(\mu_{n}\right)+\limsup _{n \rightarrow \infty} E_{n}^{(p, q ; \mathrm{F})}\left(\mu_{n}\right)+\limsup _{n \rightarrow \infty} E_{n}^{(p, q ; \mathrm{L})}\left(\mu_{n}\right) \\
& \stackrel{\text { 3.9, (2.8) }}{\leq} E^{(p, q ; \mathrm{i})}(\mu)+E^{(p, q ; \mathrm{F})}(\mu)=E^{(p, q)}(\mu)
\end{aligned}
$$

For $p=3,4$, we can repeat the same argument for $\xi \in Y_{1}^{(p)}$.

Condition (iii). As $E^{(p, q ; \mathrm{F})}$ is continuous on $\mathcal{X}_{1}$, it is sufficient to prove condition (iii) for the interaction part. If $p=1,2$, this condition is given by Lemma A.4(ii). For $p=3,4$, we use Lemma 2.6 to argue that we can split the proof by showing separately that the following three inclusions are lower energy dense:

$$
\begin{aligned}
Y_{1}^{(3)} \stackrel{(\mathrm{a})}{\subset} Y_{1}^{(4)} \stackrel{(\mathrm{b})}{\subset} X_{1} & \text { with respect to } E^{(3, q ; \mathrm{i})}, \\
Y_{1}^{(4)} \stackrel{(\mathrm{c})}{\subset} X_{1} & \text { with respect to } E^{(4, q ; \mathrm{i})} .
\end{aligned}
$$


Energy density of inclusions (b) and (c) follows from Theorem A.2 For inclusion (a), let $\xi \in Y_{1}^{(3)}$ and $\varepsilon_{n} \downarrow 0$. Take $\xi_{n}(t):=\left(1+\varepsilon_{n}\right)^{-1}\left(\xi(t)+\varepsilon_{n} t\right)$. Then

$$
\xi_{n}^{\prime}=\frac{\xi^{\prime}+\varepsilon_{n}}{1+\varepsilon_{n}} \geq \frac{\varepsilon_{n}}{1+\varepsilon_{n}}>0, \quad \sup \xi_{n} \leq 1,
$$

and hence $\left(\xi_{n}\right) \subset Y_{1}^{(3)}$. Obviously, $\xi_{n} \rightarrow \xi$ in $B V_{\text {loc }}(0,1)$, and

$$
\begin{aligned}
\limsup _{n \rightarrow \infty} E^{(3, q ; \mathrm{i})}\left(\xi_{n}\right) & =\|V\|_{L^{1}(0, \infty)} \limsup _{n \rightarrow \infty} \int_{0}^{1} \frac{1}{\xi_{n}^{\prime}} \\
& \leq\|V\|_{L^{1}(0, \infty)} \limsup _{n \rightarrow \infty} \int_{0}^{1} \frac{1+\varepsilon_{n}}{\xi^{\prime}}=E^{(3, q ; \mathrm{i})}(\xi) .
\end{aligned}
$$

Let $p=5$. As said before, we only have to regard $\mu=\left.\mathcal{L}\right|_{[0,1]}$, because $E^{(5, q)}(\mu)$ is infinite for any other $\mu$. We take the sequence $\mu_{n}$ related to $x_{i}^{n}=i / n$. Clearly $\left.\mu_{n} \rightarrow \mathcal{L}\right|_{[0,1]}$. We prove (3.1b) by explicitly calculating the limsup of all three parts of the energy.

Obviously, $E_{n}^{(5, q ; \mathrm{L})}\left(\mu_{n}\right)=0$, and

$$
E_{n}^{(5, q ; \mathrm{F})}\left(\mu_{n}\right) \stackrel{(2.8)}{\longrightarrow} E^{(5, q ; \mathrm{F})}\left(\left.\mathcal{L}\right|_{[0,1]}\right)=\frac{\beta}{2},
$$

where $\beta$ is defined in (1.12). It is exactly here that we need the condition $\beta<\infty$ as imposed in Theorem 1.1] because we need $E^{(5, q)}\left(\left.\mathcal{L}\right|_{[0,1]}\right)$ to be finite to obtain a nontrivial limit energy.

For the limsup of $E_{n}^{(5, q ; \mathrm{i})}\left(\mu_{n}\right)$, we use estimate (3.14) with $m=1$ to obtain

$$
\begin{aligned}
E_{n}^{(5, q ; \mathrm{i})}\left(\mu_{n}\right) & =\frac{\exp \left(2\left(\alpha_{n}-1\right)\right)}{n \alpha_{n}} \sum_{k=1}^{n} \sum_{j=0}^{n-k} V\left(n \alpha_{n}\left(x_{j+k}^{n}-x_{j}^{n}\right)\right) \\
& \leq \frac{2}{e^{2}}\left(1+\mathcal{O}\left(e^{-2 \alpha_{n}}\right)\right) \rightarrow \frac{2}{e^{2}} .
\end{aligned}
$$

By gathering the results above, we obtain

$$
\begin{aligned}
\limsup _{n \rightarrow \infty} E_{n}^{(5, q)}\left(\mu_{n}\right) & \leq \limsup _{n \rightarrow \infty} E_{n}^{(5, q ; \mathrm{i})}\left(\mu_{n}\right)+\limsup _{n \rightarrow \infty} E_{n}^{(5, q ; \mathrm{F})}\left(\mu_{n}\right)+\limsup _{n \rightarrow \infty} E_{n}^{(5, q ; \mathrm{L})}\left(\mu_{n}\right) \\
& \leq \frac{2}{e^{2}}+\frac{\beta}{2}=E^{(5, q)}\left(\left.\mathcal{L}\right|_{[0,1]}\right) .
\end{aligned}
$$

\section{Further results and applications}

Although the proof of Theorem 1.1 is complete, we still need to treat the special case (i.e. $p=5, q=2$ and $\beta=\infty$ (see (1.12) ) ) which is not covered by Theorem 1.1 Furthermore, we show that $E^{(p, q)}$ has a unique minimizer, which is, moreover, the limit of the sequence of minimizers of $E_{n}^{(p, q)}$.

\subsection{The particular case $p=5, q=2$ and $\beta=\infty$}

As mentioned in the introduction, the term coming from the finite domain is negligible with respect to the force term if $p=5$ and $\beta=\infty$. By considering the scaling of $\mathcal{E}$ as given by $E_{n}^{(5,2)}$, the only candidate for the $\Gamma$-limit would be $\infty$ (we do not prove 
this), which means that $E_{n}^{(5,2)}$ does not contain information in the limit. This is not unexpected, because this scaling of $\mathcal{E}$ is based on balancing the interaction term with the term coming from the finite domain. Here, we consider the scaling coming from balancing the interaction term with the force term (see (1.1)). Let $\hat{E}_{n}:=E_{n}^{(5,1)}$. Because we only consider the specific case $p=5$ and $\beta=\infty$ in this section, we do not incorporate it in the notation of $\hat{E}_{n}$, nor in its $\Gamma$-limit $\hat{E}$, which is defined by

$$
\begin{aligned}
& \hat{E}: \mathcal{P}([0, \infty)) \rightarrow \mathbb{R} \\
& \hat{E}(\mu):=\hat{E}^{(\mathrm{i})}(\mu)+\hat{E}^{(\mathrm{F})}(\mu)+\hat{E}^{(\mathrm{L})}(\mu) \\
& \hat{E}^{(\mathrm{i})}(\mu):=E^{(5,0 ; \mathrm{i})}(\mu) \\
& \hat{E}^{(\mathrm{F})}(\mu):=E^{(5,1 ; \mathrm{F})}(\mu) \\
& \hat{E}^{(\mathrm{L})}(\mu):= \begin{cases}0, & \text { if supp } \mu \subset[0, \gamma], \\
\infty, & \text { otherwise. }\end{cases}
\end{aligned}
$$

We emphasize that $\hat{E}^{(\mathrm{i})}\left(\left.\mathcal{L}\right|_{(0,1)}\right)=0$, even when $\gamma=1$. Just as before, we regard $\hat{E}$ as a mapping from $X$ to $\mathbb{R}$ whenever that is more convenient.

Theorem 4.1 (Convergence of the energy; particular case). Let $p=5, q=2$ and $\beta=\infty$, and consider $\mathcal{P}([0, \infty))$ equipped with the narrow topology. If $\left(\mu_{n}\right) \subset \mathcal{P}([0, \infty))$ is such that $\hat{E}_{n}\left(\mu_{n}\right)$ is bounded, then $\left(\mu_{n}\right)$ is compact. Moreover, $\hat{E}_{n} \Gamma$-converges to $\hat{E}$.

Proof of Theorem 4.1 The proof is similar to the proof of Theorem 1.1 In fact, the proof for the compactness statement is the same, so we do not repeat it here. The proof for the $\Gamma$-convergence again consists of proving the following two inequalities:

$$
\begin{aligned}
& \text { for all } \mu_{n} \rightarrow \mu, \\
& \text { for all } \mu \text { there exists } \mu_{n} \rightarrow \mu \text { such that } \liminf _{n \rightarrow \infty} \hat{E}_{n}\left(\mu_{n}\right) \geq \hat{E}(\mu), \\
& \limsup _{n \rightarrow \infty} \hat{E}_{n}\left(\mu_{n}\right) \leq \hat{E}(\mu),
\end{aligned}
$$

For (4.2a), note that by (3.2) we have $\liminf _{n \rightarrow \infty} \hat{E}_{n}^{(\mathrm{i})}\left(\mu_{n}\right) \geq \hat{E}^{(\mathrm{i})}(\mu)$, and by (2.7) and Proposition 2.14, we have

$$
\liminf _{n \rightarrow \infty} \hat{E}_{n}^{(\mathrm{F})}\left(\mu_{n}\right) \geq \hat{E}^{(\mathrm{F})}(\mu), \quad \liminf _{n \rightarrow \infty} \hat{E}_{n}^{(\mathrm{L})}\left(\mu_{n}\right) \geq \hat{E}^{(\mathrm{L})}(\mu) .
$$

Together these prove (4.2a $)$.

We prove (4.2b) separately for $\gamma>1$ and $\gamma=1$. In the first case, we use Theorem 2.11 to prove (4.2b) for non-decreasing functions $\xi$. We can restrict ourselves to proving (4.2b) only for $\xi \in X_{\gamma}$; for these $\xi, \hat{E}^{(\mathrm{L})}(\xi)=0$. The subscript in the notation for $X$ refers to the upper bound for $\sup \xi$, just as it did in the spaces defined by (3.16). By Proposition 2.12, this upper bound on $\xi$ implies that the force term is a continuous perturbation to $\hat{E}_{n}$, so by Theorem B.3 it is enough to prove

for all $\xi \in X_{\gamma}$ there exists $\xi_{n} \rightarrow \xi$ in $B V_{\text {loc }}(0,1)$ such that

$$
\limsup _{n \rightarrow \infty} \hat{E}_{n}^{(\mathrm{i})}\left(\xi_{n}\right)+\hat{E}_{n}^{(\mathrm{L})}\left(\xi_{n}\right) \leq \hat{E}^{(\mathrm{i})}(\xi),
$$


We prove (4.3) by applying Lemma 2.4 with the subset

$$
Z_{\gamma}^{(5)}:=\left\{\xi \in Y_{\gamma}^{(5)} \mid \xi(1)<\gamma\right\} .
$$

This requires its two conditions to be satisfied:

Condition (ii). Let $\xi \in Z_{\gamma}^{(5)}$ and take $\left(\xi_{n}\right) \subset X_{\gamma}$ as defined by (3.7). Note that $\sup \xi_{n} \rightarrow \sup \xi<\gamma$, which together with $\gamma_{n} \rightarrow \gamma$ implies that indeed $\left(\xi_{n}\right) \subset X_{\gamma}$ for all $n$ large enough. Furthermore, we have $\sup \xi_{n} \leq \gamma_{n}$ for all $n$ large enough, which implies $\hat{E}_{n}^{(\mathrm{L})}\left(\xi_{n}\right)=0$. Hence

$$
\limsup _{n \rightarrow \infty} \hat{E}_{n}^{(\mathrm{i})}\left(\xi_{n}\right)+\hat{E}_{n}^{(\mathrm{L})}\left(\xi_{n}\right)=\limsup _{n \rightarrow \infty} E_{n}^{(5,0 ; \mathrm{i})}\left(\xi_{n}\right) \stackrel{\sqrt[3.9]{\leq}}{\leq} E^{(5,0 ; \mathrm{i})}(\xi) .
$$

Condition (iii). By Lemma 2.6 it is enough to show that the following three inclusions are energy dense:

$$
Z_{\gamma}^{(5)} \subset Y_{\gamma}^{(4)} \subset X_{\gamma} \text { with respect to } \hat{E}^{(\mathrm{i})} .
$$

Energy density of the second inclusion follows from Theorem A.2. To show the first inclusion, we take $\xi \in Y_{\gamma}^{(4)}$. This implies that $\xi \in W_{\text {incr }}^{1,1}, \xi(1) \leq \gamma$ and $\inf \xi^{\prime} \geq 1$. It is enough to construct $\xi_{n} \rightarrow \xi$ in $B V_{\text {loc }}$ such that $\xi_{n} \in W_{\text {incr }}^{1,1}, \xi_{n}(1)<\gamma$ and inf $\xi_{n}^{\prime}>1$, because then $\left(\xi_{n}\right) \subset Z_{\gamma}^{(5)}$ and $\hat{E}_{n}^{(\mathrm{i})}\left(\xi_{n}\right)+\hat{E}_{n}^{(\mathrm{L})}\left(\xi_{n}\right)=0$. It is easy to see that $\xi_{n}$ as defined by

$$
\xi_{n}(t):=\frac{1-\varepsilon_{n}}{1+\varepsilon_{n}}\left(\xi(t)+\varepsilon_{n} \gamma t\right)+\varepsilon_{n} t
$$

for some $\varepsilon_{n} \downarrow 0$, satisfies all these requirements. Note that the strict inequalities in the requirements for $\xi_{n}$ are obtained solely by using $\gamma>1$. This completes the proof for (4.2b) under the assumption that $\gamma>1$.

We now turn to the case $\gamma=1$. As the following proof is similar to the proof of Theorem 1.1 in case $p=5$ and $q=2,3$ (see page 19), we do this in terms of measures instead of using non-decreasing functions. Again, we have that $\hat{E}(\mu)$ can only be finite when $\mu=\left.\mathcal{L}\right|_{(0,1)}$, but now we take $\mu_{n}$ as defined by $x_{i}^{n}:=\gamma_{n} i / n$. This is to ensure that $\hat{E}_{n}^{(\mathrm{L})}\left(\mu_{n}\right)=0$. Clearly $\hat{E}_{n}^{(\mathrm{F})}\left(\mu_{n}\right) \rightarrow 1 / 2=\hat{E}^{(\mathrm{F})}\left(\left.\mathcal{L}\right|_{(0,1)}\right)$, so it is only left to prove that $\hat{E}_{n}^{(\mathrm{i})}\left(\mu_{n}\right) \rightarrow 0$. Due to $x_{i}^{n}=\gamma_{n} i / n$, we get

$$
\sum_{k=1}^{n} \sum_{j=0}^{n-k} V\left(n \hat{\alpha}_{n}\left(x_{j+k}^{n}-x_{j}^{n}\right)\right) \stackrel{(3.14)}{\leq} 2 n \hat{\alpha}_{n} \gamma_{n} e^{-2 \gamma_{n} \hat{\alpha}_{n}}+\mathcal{O}\left(e^{-4 \gamma_{n} \hat{\alpha}_{n}}\right),
$$

and hence

$$
\begin{aligned}
\hat{E}_{n}^{(\mathrm{i})}\left(\mu_{n}\right) & =\frac{\exp \left(2\left(\hat{\alpha}_{n}-1\right)\right)}{n \hat{\alpha}_{n}} \sum_{k=1}^{n} \sum_{j=0}^{n-k} V\left(n \hat{\alpha}_{n}\left(x_{j+k}^{n}-x_{j}^{n}\right)\right) \\
& \leq \frac{2}{e^{2}} \gamma_{n}\left(e^{2 \hat{\alpha}_{n}\left(1-\gamma_{n}\right)}+\mathcal{O}\left(e^{-2 \hat{\alpha}_{n}}\right)\right) \\
& \leq \frac{1.8}{e^{2}} \gamma_{n}\left(e^{2 \alpha_{n}\left(1 / \gamma_{n}-1\right)}+\mathcal{O}\left(e^{-2 \hat{\alpha}_{n}}\right)\right) \rightarrow 0,
\end{aligned}
$$

in which the convergence to 0 follows from $\beta=\infty$. 


\subsection{Existence, uniqueness and convergence of minimizers}

Theorem 4.2 (Existence and uniqueness of minimizers). Let $p \in\{1, \ldots, 5\}, q \in\{0, \ldots, 3\}$. The minimization problem

$$
\min _{\mu \in \mathcal{P}([0, \infty))} E^{(p, q)}(\mu)
$$

has a unique minimizer. The energy $\hat{E}$ (as defined in (4.1)) has a unique minimizer as well.

Proof of Theorem 4.2 For case $q=0$, this has been proved in ([15], Theorem 2). Because our proofs for $q=1,2,3$ are similar to that proof, we state the intermediate results of that proof first.

To show existence, take a minimizing sequence $\left(\mu_{m}\right)_{m \in \mathbb{N}}$. Since for each of the limit energies either $E^{(p, q ; \mathrm{F})}$ or $E^{(p, q ; \mathrm{L})}$ is non-vanishing, $\left(\mu_{m}\right)$ is tight, and therefore narrowly compact. Since each of the terms in the limiting energies is narrowly lower semicontinuous, existence follows.

To show uniqueness, note that $E^{(\mathrm{F})}$ and $E^{(\mathrm{L})}$ are convex, both in the classical sense, i.e. in the additive structure on $\mathcal{P}([0, \infty))$, and displacement convex. In [15] it was shown that $E^{(p,(0-3) ; i)}$ is strictly convex in the classical sense for $p=1,2,3$ and strictly geodesically convex for $p=4$. For all $p \leq 4$, therefore, $E^{(p,(0-3))}$ is strictly convex in some sense and therefore has exactly one minimizer. If $p=5$, it is obvious from $(2.2)$ that $\left.\mathcal{L}\right|_{[0,1]}$ is the unique minimizer of $E^{(5, q ; \mathrm{F})}$ when $\beta<\infty$. If $\beta=\infty$ (the case of Theorem 4.1), the limit energy is given by $\hat{E}(\mu)=E^{(5,0)}(\mu)+\chi_{\{\operatorname{supp} \mu \subset[0, \gamma]\}}$, for which $\left.\mathcal{L}\right|_{[0,1]}$ is the unique minimizer.

Corollary 4.3 (Convergence of minimizers). For each $n \in \mathbb{N}_{+}$, let $\mu_{n}^{*}$ and $\mu^{*}$ be the minimizers of respectively $E_{n}^{(p, q)}$ and $E^{(p, q)}$ (or $\hat{E}_{n}$ and $\hat{E}$ whenever $p=5$ and $\beta=\infty$ ). Then $\mu_{n}^{*} \rightarrow \mu^{*}$.

Proof of Corollary 4.3 The proof is the same for $p=5$ and $\beta=\infty$ as for the other cases. Hence we restrict ourselves to the other cases, and so we use the energies $E_{n}^{(p, q)}$ and $E^{(p, q)}$.

By Theorem 1.1 the sequence $\left(\mu_{n}^{*}\right)$ is narrowly compact, and converges along a subsequence to a limit $\mu$. By standard properties of $\Gamma$-convergence, $\mu$ is a minimizer of $E^{(p, q)}$. Since minimizers of $E^{(p, q)}$ are unique by Theorem 4.2 , the whole sequence converges.

\subsection{Rescaling $E_{n}^{(5,(2-3))}$}

As mentioned in Section 1.3, the $\Gamma$-limit of $E_{n}^{(5,(2-3))}$ is unsatisfactory, because it only contains information about the unique minimizer. One way to keep more information in the limit, is to consider a logarithmic scaling. More precisely, we define

$$
\mathfrak{E}_{n}\left(\mu_{n}\right):=\frac{1}{2 \alpha_{n}} \log E_{n}^{(5,(2-3))}\left(\mu_{n}\right)
$$


and show that it $\Gamma$-converges to $\mathfrak{E}$, which is given by

$$
\mathfrak{E}(\mu)= \begin{cases}1-\frac{1}{M}, & \text { if } \operatorname{supp} \mu \subset[0,1], \\ \infty, & \text { otherwise }\end{cases}
$$

where

$$
M:=\sup _{a<b} \frac{\mu((a, b))}{b-a} .
$$

We can also express $\mathfrak{E}$ in terms of non-decreasing functions as

$$
\mathfrak{E}(\xi)= \begin{cases}1-m_{\xi}, & \text { if } \sup \xi \leq 1 \\ \infty, & \text { otherwise }\end{cases}
$$

where

$$
m_{\xi}:=\inf _{a<b} \frac{D \xi((a, b))}{b-a},
$$

and $D \xi$ is the distributional derivative of $\xi$.

Theorem 4.4 ( $\Gamma$-Convergence of the logarithm of the energy). Let $p=5, q=2$ and $\beta=\infty$, and consider $\mathcal{P}([0, \infty))$ equipped with the narrow topology. If $\left(\mu_{n}\right) \subset \mathcal{P}([0, \infty))$ is such that $\hat{E}_{n}\left(\mu_{n}\right)$ is bounded, then $\left(\mu_{n}\right)$ is precompact. Moreover, $\hat{E}_{n} \Gamma$-converges to $\hat{E}$.

\section{Proof of Theorem 4.4}

The structure of the proof is similar to the $\Gamma$-convergence proof of $E_{n}^{(5,(2-3))}$. Compactness follows from the same argument as used for showing compactness for $E_{n}^{(5,(2-3))}$, because we still require for any fixed $n$ that $E_{n}^{(5,(2-3) ; \mathrm{L})}\left(\mu_{n}\right)<\infty$ in order for $\mathfrak{E}_{n}\left(\mu_{n}\right)$ to be finite.

To show the liminf inequality, we separate three cases: $\mu=\left.\mathcal{L}\right|_{(0,1)}$, supp $\mu \nsubseteq[0,1]$, and all other $\mu \in \mathcal{P}([0, \infty))$.

If $\mu=\left.\mathcal{L}\right|_{(0,1)}$, we see from (3.11) for any $\mu_{n} \rightarrow \mu$ that

$$
\liminf _{n \rightarrow \infty} \mathfrak{E}_{n}\left(\mu_{n}\right) \geq \liminf _{n \rightarrow \infty} \frac{1}{2 \alpha_{n}} \log \left(2 e^{-2}+\mathcal{O}\left(e^{-2 \alpha_{n}}\right)\right)=0 .
$$

If $\operatorname{supp} \mu \nsubseteq[0,1]$, it follows from lower semi-continuity (see (2.7c) and Proposition 2.14) that $E_{n}^{(5,(2-3) ; \mathrm{L})}\left(\mu_{n}\right)=\infty$ for $n$ large enough for any $\mu_{n} \rightarrow \mu$, so that $\mathfrak{E}_{n}\left(\mu_{n}\right)=\infty$ as well.

If $\operatorname{supp} \mu \subset[0,1]$ and $\mu \neq\left.\mathcal{L}\right|_{(0,1)}$, we have that $1<M$. As we like to have explicit values for $a, b$ in the calculation below (rather than the supremum over them as in the definition of $M)$, we fix $0<\varepsilon<M-1$, and take $a_{\varepsilon}<b_{\varepsilon}$ such that $\left(b_{\varepsilon}-a_{\varepsilon}\right)^{-1} \mu\left(\left(a_{\varepsilon}, b_{\varepsilon}\right)\right)>$ $M-\varepsilon=: M_{\varepsilon}$. We follow the same reasoning as for (3.12) to find

$$
\begin{aligned}
\liminf _{n \rightarrow \infty} \mathfrak{E}_{n}\left(\mu_{n}\right) & \geq \liminf _{n \rightarrow \infty} \frac{1}{2 \alpha_{n}} \log \left(2 e^{-2}\left(b_{\varepsilon}-a_{\varepsilon}\right) \exp \left(2 \alpha_{n}\left(1-M_{\varepsilon}^{-1}\right)\right)\left(1+\mathcal{O}\left(e^{-2 \alpha_{n} / M_{\varepsilon}}\right)\right)\right) \\
& =\liminf _{n \rightarrow \infty}\left(1-(M-\varepsilon)^{-1}+O\left(\alpha_{n}^{-1}\right)\right)=1-(M-\varepsilon)^{-1} .
\end{aligned}
$$

Since $\varepsilon$ was chosen arbitrarily, we obtain

$$
\liminf _{n \rightarrow \infty} \mathfrak{E}_{n}\left(\mu_{n}\right) \geq 1-\frac{1}{M}
$$


We continue with the proof of the limsup inequality. We can restrict to $\xi \in X_{1}$, because otherwise $\mathfrak{E}$ is infinite. We conclude the limsup inequality from Lemma 2.4 after showing that its two conditions are satisfied. We use Lemma 2.4 with the subset $X_{1}^{(5)}:=\left\{\xi \in X_{1} \mid m_{\xi}>0\right\}$.

Condition (ii). Let $\xi \in X_{1}^{(5)}$. We construct $\xi_{n}$ by using linear interpolation (see (2.5b) with $x_{i}^{n}:=\sup _{t<i / n} \xi(t)$ (because $\xi$ need not be in $W^{1,1}, \xi$ can not be evaluated at specific values). From Proposition 2.12 we conclude that for $n$ large enough it holds

$$
E_{n}^{(5,(2-3) ; \mathrm{F})}\left(\xi_{n}\right)<E^{(5,(2-3) ; \mathrm{F})}(\xi)+1 \leq \frac{\beta}{2}+1 .
$$

Observe that for any $i, j \in\{0, \ldots, n\}$ with $i>j$, we have the estimate

$$
\left(x_{i}^{n}-x_{j}^{n}\right)=D \xi([j / n, i / n)) \geq m_{\xi} \frac{i-j}{n} .
$$

This is a similar estimate as (3.13). This allows us to use (3.14) to derive the following upper bound

$$
\begin{aligned}
E_{n}^{(5,(2-3) ; \mathrm{i})}\left(\xi_{n}\right) & =\frac{\exp \left(2\left(\alpha_{n}-1\right)\right)}{n \alpha_{n}} \sum_{k=1}^{n} \sum_{j=0}^{n-k} V\left(n \alpha_{n}\left(x_{j+k}^{n}-x_{j}^{n}\right)\right) \\
& \leq \frac{2 m_{\xi}}{e^{2}} e^{2 \alpha_{n}\left(1-m_{\xi}\right)}\left(1+\mathcal{O}\left(e^{-2 m_{\xi} \alpha_{n}}\right)\right) \rightarrow 0 .
\end{aligned}
$$

By combining the estimates on $E_{n}^{(5,(2-3) ; \mathrm{i})}\left(\xi_{n}\right)$ and $E_{n}^{(5,(2-3) ; \mathrm{F})}\left(\xi_{n}\right)$ we obtain

$$
\begin{aligned}
\limsup _{n \rightarrow \infty} \mathfrak{E}_{n}\left(\xi_{n}\right) & =\limsup _{n \rightarrow \infty} \frac{1}{2 \alpha_{n}} \log \left(E_{n}^{(5,(2-3) ; \mathrm{i})}\left(\xi_{n}\right)+E_{n}^{(5,(2-3) ; \mathrm{F})}\left(\xi_{n}\right)\right) \\
& \leq \limsup _{n \rightarrow \infty} \frac{1}{2 \alpha_{n}} \log \left(\frac{2 m_{\xi}}{e^{2}} e^{2 \alpha_{n}\left(1-m_{\xi}\right)}\left(1+\mathcal{O}\left(e^{-2 m_{\xi} \alpha_{n}}\right)\right)+\frac{\beta}{2}+1\right) \\
& =1-m_{\xi}=\mathfrak{E}(\xi) .
\end{aligned}
$$

Condition (iii). Let $\xi \in X_{1}$, and define

$$
\xi_{n}(t):=\frac{\xi(t)+t \varepsilon_{n}}{1+\varepsilon_{n}}
$$

for some $\varepsilon_{n} \downarrow 0$. By construction, $\xi_{n} \in X_{1}^{(5)}$, which follows from

$$
\begin{gathered}
\sup \xi_{n}=\frac{\sup \xi+\varepsilon_{n}}{1+\varepsilon_{n}} \leq 1, \quad \text { and } \\
m_{\xi_{n}}=\inf _{b>a} \frac{D \xi((a, b))+\varepsilon_{n} \mathcal{L}((a, b))}{b-a}=m_{\xi}+\varepsilon_{n}>0 .
\end{gathered}
$$

Clearly $\xi_{n} \rightarrow \xi$ in $B V_{\mathrm{loc}}(0,1)$, and

$$
\mathfrak{E}\left(\xi_{n}\right)=1-m_{\xi_{n}}=1-\left(m_{\xi}+\varepsilon_{n}\right) \rightarrow \mathfrak{E}(\xi) .
$$




\section{Acknowledgements}

We would like to thank M. Geers, R. Peerlings, M. Hütter, M. Kooiman and M. Dogge for fruitful discussions which have led us to our microscopic model, and L. Scardia for many discussions throughout this project.

PvM kindly acknowledges the financial support from the Netherlands Organisation for Scientific Research (NWO). He is part of the CorFlux project (nr. 10012310), which is one of the fourteen projects in the complexity program of NWO.

\section{Appendix A Technical steps}

Lemma A.1 (A support property of narrow convergence). Let $\mu_{n}, \mu \in \mathcal{P}([0, \infty))$ and $a_{n}, a \in \mathbb{R}$ such that $\mu_{n} \rightarrow \mu$ and $a_{n} \rightarrow a$. If $\operatorname{supp} \mu \nsubseteq[0, a]$, then $\operatorname{supp} \mu_{n} \nsubseteq\left[0, a_{n}\right]$ for all $n$ large enough.

Proof of Lemma A.1 The proof goes by contradiction. Suppose there exists a subsequence $\left(\mu_{n}\right)$ such that $\operatorname{supp} \mu_{n} \subset\left[0, a_{n}\right]$. supp $\mu \nsubseteq[0, a]$ and inner regularity imply that there is a closed interval $K$ in $(a, \infty)$ such that $\mu(K)>0$. It is straightforward to choose a test function $\varphi \in C_{b}([0, \infty))$ such that

$$
\int_{0}^{\infty} \varphi d \mu_{n}=0
$$

for $n$ large enough, and

$$
\int_{0}^{\infty} \varphi d \mu>0
$$

which contradicts with $\mu_{n} \rightarrow \mu$.

The following theorem is a generalization of [15, Theorem 4], in the sense that it applies to the sets $X_{\gamma}$ and $Y_{\gamma}^{(4)}$ (see (3.16)) not only for $\gamma=\infty$, but also for any $\gamma \in(0, \infty)$. The proof in [15] holds for finite $\gamma$ as well.

Theorem A.2 (A sufficient condition for energy density). Let $f:(0, \infty) \rightarrow \overline{\mathbb{R}}$ be convex and decreasing, such that $\lim _{t \rightarrow \infty} f(t)=0$. Let $E: X_{\gamma} \rightarrow \overline{\mathbb{R}}$,

$$
E(u):=\int_{0}^{1} f\left(u^{\prime}(t)\right) d t .
$$

Then $Y_{\gamma}^{(4)}$ is energy dense in $X_{\gamma}$ with respect to $E$.

Remark A.3 Just as in [15, we use Theorem A.2 for $E^{(p, q ; i)}$ for $p=3,4,5$ and $q=$ $0, \ldots, 3$. In these cases, we take for $f(t)$ respectively

$$
\frac{1}{t}, \quad \sum_{k=1}^{\infty} V(k t), \quad \chi_{\{t \geq 1\}}
$$

Lemma A.4 (Energy density results). Let $p \in\{1,2\}$, and $\mathcal{X}_{1}$ and $\mathcal{Y}_{1}$ as defined by (3.16). Then 
(i) $\mathcal{Y}$ is energy dense in $\mathcal{P}([0, \infty))$ with respect to $E^{(p, q)}$ for $q=0,1$.

(ii) $\mathcal{Y}_{1}$ is energy dense in $\mathcal{X}_{1}$ with respect to $E^{(p, q ; i)}$ for $q=2,3$.

Proof of Lemma A.4 Lemma A.4, (i) is proved by using Lemma 2.6. It involves the intermediate space

$$
\tilde{\mathcal{Y}}:=\left\{\mu \in \mathcal{P}([0, \infty)) \mid \mu \ll \mathcal{L}, \frac{d \mu}{d \mathcal{L}} \in L^{\infty}(0, \infty)\right\} .
$$

The proof of $\tilde{\mathcal{Y}}$ being energy dense in $\mathcal{P}([0, \infty))$ is stated in [15, proof of limsup inequality Theorem 5]. The related sequence of the limsup inequality is given by the measures corresponding to the densities given by

$$
\rho_{n}(x):=n \mu([x, x+1 / n)) .
$$

Still to be shown: $\forall \mu \in \tilde{\mathcal{Y}} \exists\left(\mu_{n}\right) \subset \mathcal{Y}: \mu_{n} \rightarrow \mu$, and $\limsup _{n \rightarrow \infty} E^{(p, 0)}\left(\mu_{n}\right) \leq E^{(p, 0)}(\mu)$.

Let $\rho:=\frac{d \mu}{d \mathcal{L}}$, and take $\rho_{n}=\frac{1}{\mu([0, n])} \rho \mathbb{1}_{[0, n]}$. For the related $\mu_{n}$, it is easy to see that $\mu_{n} \rightarrow \mu$ and that $E^{(p, 0 ; \mathrm{F})}\left(\mu_{n}\right) \leq E^{(p, 0 ; \mathrm{F})}(\mu)$. By using the Dominated Convergence Theorem, one can prove $E^{(p, 0 ; \mathrm{i})}\left(\mu_{n}\right) \rightarrow E^{(p, 0 ; \mathrm{i})}(\mu)$.

The proof above works just as well for proving Lemma A.4,(iii), because we can identify $\left.E^{(p,(2-3) ; \mathrm{i})}\right|_{\mathcal{X}_{1}}$ by $E^{(p, 0 ; \mathrm{i})} \mid \mathcal{X}_{1}$, since $\hat{\alpha}_{n}$ and $\alpha_{n}$ play the same role.

\section{Appendix B $\Gamma$-convergence}

Here, we state the basic properties of $\Gamma$-convergence, which can be found, for example, in [26]. Although $\Gamma$-convergence can be defined on topological spaces, we only show the definition for metric spaces:

Definition B.1 (Г-convergence). Let $(X, d)$ be a metric space, $E_{n}, E: X \rightarrow \overline{\mathbb{R}}$. Then $E_{n}$ $\Gamma$-converges to $E$ with respect to $d$ iff the following two conditions are satisfied:

(i) $\forall x \in X \forall x_{n} \stackrel{d}{\rightarrow} x: \liminf _{n \rightarrow \infty} E_{n}\left(x_{n}\right) \geq E(x)$,

(ii) $\forall x \in X \exists y_{n} \stackrel{d}{\rightarrow} x: \limsup _{n \rightarrow \infty} E_{n}\left(y_{n}\right) \leq E(x)$.

The sequence $\left(y_{n}\right)$, if it exists, is called the recovery sequence.

We continue by stating some properties of $\Gamma$-convergence that are useful to us. Let $(X, d)$ be a metric space, and $E_{n}, E: X \rightarrow \overline{\mathbb{R}}$. The next Theorem [26, Proposition 6.20] states one of the most important properties of $\Gamma$-convergence. We need the following definition first:

Definition B.2 (Continuous convergence). Let $F_{n}, F: X \rightarrow \mathbb{R}$. Then $F_{n} \rightarrow F$ continuously iff

$$
\forall x \in X: \lim _{\varepsilon \rightarrow 0} \limsup _{n \rightarrow \infty} \sup _{y \in B(x, \varepsilon)}\left|F_{n}(y)-F(x)\right|=0 .
$$

Theorem B.3 (Stability of $\Gamma$-convergence under continuously converging perturbations). 
Let $F_{n}, F: X \rightarrow \mathbb{R}$. If $F_{n} \rightarrow F$ continuously, then

$$
E_{n}+F_{n} \stackrel{\Gamma}{\rightarrow} E+F .
$$

Remark B.4 Note that $R(F) \subset \mathbb{R} \subsetneq \overline{\mathbb{R}}$ is required in Definition B.2.

\section{References}

[1] S. Cacace and A. Garroni. A multi-phase transition model for dislocations with interfacial microstructure. Interfaces Free Bound, 11:291-316, 2009.

[2] W. Cai, A. Arsenlis, C.R. Weinberger, and V.V. Bulatov. A non-singular continuum theory of dislocations. Journal of the Mechanics and Physics of Solids, 54(3):561-587, 2006.

[3] W.D. Callister. Materials Science and Engineering, An Introduction. John Wiley \& Sons, 2007.

[4] P. Cermelli and G. Leoni. Renormalized energy and forces on dislocations. SIAM Journal on Mathematical Analysis, 37(4):1131-1160, 2006.

[5] J. Deng and A. El-Azab. Dislocation pair correlations from dislocation dynamics simulations. Journal of Computer-Aided Materials Design, 14(1):295-307, 2007.

[6] J. Deng and A. El-Azab. Mathematical and computational modelling of correlations in dislocation dynamics. Modelling and Simulation in Materials Science and Engineering, 17:075010, 2009.

[7] M. Dogge. Mechanics of Phase Boundaries. PhD thesis, Eindhoven University of Technology, To appear...

[8] M.H. Duong, V. Laschos, and M. Renger. Wasserstein gradient flows from large deviations of thermodynamic limits. arXiv: 1203.0676v2, 2012.

[9] L.P. Evers, W.A.M. Brekelmans, and M.G.D. Geers. Scale dependent crystal plasticity framework with dislocation density and grain boundary effects. International Journal of Solids and Structures, 41(18-19):5209-5230, 2004.

[10] M. Focardi and A. Garroni. A 1D macroscopic phase field model for dislocations and a second order Г-limit. Multiscale Modeling \& Simulation, 6(4):1098-1124, 2007.

[11] N. Forcadel, C. Imbert, and R. Monneau. On the Notions of Solutions to Nonlinear Elliptic Problems: Results and Developments, chapter Viscosity solutions for particle systems and homogenization of dislocation dynamics. Department of Mathematics of the Seconda Universita di Napoli, 2008.

[12] N. Forcadel, C. Imbert, and R. Monneau. Homogenization of the dislocation dynamics and of some particle systems with two-body interactions. Discrete and Continuous Dynamical Systems A, 23(3):785-826, 2009.

[13] A. Garroni, G. Leoni, and M. Ponsiglione. Gradient theory for plasticity via homogenization of discrete dislocations. arXiv: 0808.2361, 2008.

[14] A. Garroni and S. Müller. Г-limit of a phase-field model of dislocations. SIAM Journal on Mathematical Analysis, 36(6):1943-1964, 2005.

[15] M.G.D. Geers, R.H.J. Peerlings, M.A. Peletier, and L. Scardia. Asymptotic behaviour of a pile-up of infinite walls of edge dislocations. Archive for Rational Mechanics and Analysis, 209:495-539, 2013.

[16] I. Groma. Link between the microscopic and mesoscopic length-scale description of the collective behavior of dislocations. Physical Review B, 56(10):5807-5813, 1997.

[17] I. Groma, F.F. Csikor, and M. Zaiser. Spatial correlations and higher-order gradient terms in a continuum description of dislocation dynamics. Acta Materialia, 51(5):1271-1281, 2003.

[18] A. El Hajj, H. Ibrahim, and R. Monneau. Homogenization of dislocation dynamics. In IOP Conferences Series: Materials Science and Engineering, 2009. 
[19] C.L. Hall. Asymptotic expressions for the nearest and furthest dislocations in a pile-up against a grain boundary. Philosophical Magazine, 90(29):3879-3890, 2010.

[20] C.L. Hall. Asymptotic analysis of a pile-up of regular edge dislocation walls. Materials Science and Engineering: A, 530:144-148, 2011.

[21] C.L. Hall, S.J. Chapman, and J.R. Ockendon. Asymptotic analysis of a system of algebraic equations arising in dislocation theory. SIAM Journal on Applied Mathematics, 70(7):2729-2749, 2010.

[22] J.P. Hirth and J. Lothe. Theory of Dislocations. John Wiley \\& Sons, 1982.

[23] D. Hull and D.J. Bacon. Introduction to Dislocations. Oxford : Butterworth Heinemann, 2001.

[24] M. Koslowski and M. Ortiz. A multi-phase field model of planar dislocation networks. Modelling and Simulation in Materials Science and Engineering, 12(6):1087, 2004.

[25] S. Limkumnerd and E. Van der Giessen. Statistical approach to dislocation dynamics: From dislocation correlations to a multiple-slip continuum theory of plasticity. Physical Review $B, 77(18): 184111,2008$.

[26] G. Dal Maso. An Introduction to Г-Convergence. Birkhäuser Boston, 1993.

[27] M. Ponsiglione. Elastic energy stored in a crystal induced by screw dislocations: from discrete to continuous. SIAM Journal on Mathematical Analysis, 39(2):449-469, 2007.

[28] J. Portegies. Non-equidistant dislocation walls. Technical report, Eindhoven University of Technology, 2013. To appear...

[29] A. Roy, R.H.J. Peerlings, M.G.D. Geers, and Y. Kasyanyuk. Continuum modeling of dislocation interactions: Why discreteness matters? Materials Science and Engineering: A, 486:653-661, 2008.

[30] L. Scardia, R.H.J. Peerlings, M.A. Peletier, and M.G.D. Geers. Mechanics of dislocation pile-ups: a unification of scaling regimes. Journal of the Mechanics and Physics of Solids. to appear...

[31] S. Serfay. Gamma-convergence of gradient flows on hilbert and metric spaces and applications. Discrete and Continuous Dynamical Systems A, 31:1427-1451, 2011.

[32] R.E. Voskoboinikov, S.J. Chapman, J.B. McLeod, and J.R. Ockendon. Asymptotics of edge dislocation pile-up against a bimetallic interface. Mathematics and Mechanics of Solids, 14:284-295, 2009.

[33] S. Yefimov, I. Groma, and E. Van der Giessen. A comparison of a statistical-mechanics based plasticity model with discrete dislocation plasticity calculations. Journal of the Mechanics and Physics of Solids, 52(2):279-300, 2004.

[34] M. Zaiser and I. Groma. Some limitations of dislocation walls as models for plastic boundary layers. arXiv: 1109.2216, 2011.

[35] M. Zaiser, M.C. Miguel, and I. Groma. Statistical dynamics of dislocation systems: The influence of dislocation-dislocation correlations. Physical Review B, 64(22):224102, 2001. 